\title{
Synthesis of 3,4,5-Trisubstituted Isoxazoles through Gold-Catalyzed Cascade Cyclization-Oxidative Alkynylation and Cyclization-Fluorination of 2-Alkynone $O$-Methyloximes
}

\author{
Doo-Hee Song and Jae-Sang Ryu* \\ College of Pharmacy \& Graduate School of Pharmaceutical Sciences, Global Top5 Research Program, \\ EwhaWomans University, Seoul 120-750,Korea. E-mail: ryuj@ewha.ac.kr \\ Received April 11, 2014, Accepted May 6, 2014
}

\begin{abstract}
Gold-catalyzed tandem cyclization-oxidative alkynylation and cyclization-fluorination reactions of 2alkynone $O$-methyloximes are described. The reactions proceed smoothly at room temperature in the presence of $10 \mathrm{~mol} \%$ of $\left(\mathrm{PPh}_{3}\right) \mathrm{AuNTf}_{2}, 2.5$ equivalents of selectfluor, and 2 equivalents of $\mathrm{K}_{3} \mathrm{PO}_{4}$. 2-Alkynone $O$ methyloximes undergo intramolecular oxyauration/cyclization and ensuing oxidative cross-coupling and fluorination process to afford the corresponding 3,4,5-trisubstituted isoxazoles in a cascade manner.
\end{abstract}

Key Words : Gold, Isoxazole, $O$-Methyloxime, Cross-coupling, Alkyne

\section{Introduction}

Isoxazole $^{1}$ is an important scaffold frequently found in many natural products and biologically active compounds such as analgesics, ${ }^{2}$ antibiotics, ${ }^{3}$ antidepressants, ${ }^{4}$ and anticancer agent. ${ }^{5}$ It has also been used as a synthetic intermediate, building block and chiral ligand in organic synthesis. ${ }^{5}$ The prevailing methods for the synthesis of isoxazoles mostly involve the condensation between a hydroxylamine and a 1,3-dicarbonyl compound, ${ }^{6}$ the condensation between a hydroxylamine and $\alpha, \beta$-unsaturated carbonyl compound, ${ }^{7}$ and the $[3+2]$ cycloaddition reaction between an alkyne and a nitrile oxide. ${ }^{8}$ These methods usually require harsh conditions and suffer from poor chemo- and regioselectivities. Furthermore, they are unsuitable for the selective synthesis of fully substituted isoxazoles. So far, only a few methodologies for the selective synthesis of fully substituted isoxazoles have been reported. Larock and co-workers reported a stepwise synthesis of trisubstituted isoxazoles that featured the iodocyclization of 2-alkynone $O$-methyloximes and a subsequent palladium-catalyzed coupling reaction. ${ }^{9}$ Harrity and co-workers demonstrated a stepwise reaction involving [3+2] cycloaddition reaction of nitrile oxides and alkynylboronates to provide isoxazole boronic esters, followed by Suzuki coupling reaction. ${ }^{10}$ Denmark and co-workers also reported a $[3+2]$ cycloaddition reaction between alkynyldimethylsilyl ethers and nitrile oxides to produce isoxazolylsilanols, the cross-coupling reactions of which afforded 3,4,5-trisubstituted isoxazoles. ${ }^{11}$ In these three cases, extra functionalization at the 4-position, such as iodination, borylation, or silylation, is required and is followed by crosscoupling reaction with a genuine substituent in a stepwise manner. Considering "atom economy" atoms and "pot economy"13 to target the inherent efficiency by avoiding intermediate isolation, the direct synthesis of trisubstituted isoxazoles in one sequence is highly desirable and challenging. However, cycloaddition reactions with internal alkynes to afford trisubstituted isoxazoles are limited to a handful of highly activated alkynes (e.g., acetylene dicarboxylate and related electron-deficient acetylenes) and suffer from poor regioselectivities ${ }^{14}$ as mentioned previously. Facile one-pot domino reactions have rarely been reported. ${ }^{15}$ The lack of direct synthetic methods for the fully substituted isoxazoles has become more acute due to increasing needs and applications in the pharmaceutical industry.

In recent years, gold catalysts have drawn significant attention due to their applicability in many organic transformations, which originates from their promising properties such as air- and moisture-stability, a broad range of functional group compatibilities, superior $\pi$-acidity, fine-tunable reactivity with various ligands, and mild reaction conditions. ${ }^{16}$ Interestingly, gold rarely changes oxidation state during the reaction. Hence, for a long time, gold-catalyzed cross-coupling reactions were not considered feasible although many organic transformations are catalyzed by gold. However, more recently, it was discovered that gold was able to undergo $\mathrm{Au}(\mathrm{I}) / \mathrm{Au}(\mathrm{III})$ redox cycles in the presence of an external oxidant such as Selectfluor, which enabled crosscoupling reactions. ${ }^{17}$ Therefore, to develop novel direct synthetic methods for the fully substituted isoxazoles, we envisioned cyclization of 2-alkynone $O$-methyloxime 1a catalyzed by $\pi$-acidic gold, leading to the cationic gold intermediate $\mathbf{2}$; the subsequent oxidative alkynylation might provide trisubstituted isoxazole $\mathbf{4}$ in a cascade manner

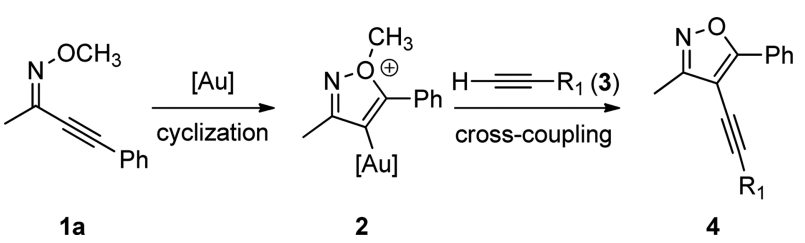

Scheme 1. Strategy for cascade cyclization-oxidative alkynylation. 
(Scheme 1). This "pot economic" transformation involves gold-catalyzed cyclization reaction combined with oxidative cross-coupling in one-pot, which allows direct access to the highly functionalized isoxazoles.

\section{Experimental}

General Remarks. Reagents were purchased in SigmaAldrich Ltd. Alfa Aesar, Strem chemicals Inc. or Acros organics, and used without further purification. All solvents were distilled before used under dry Ar over appropriate drying agents (sodium benzophenone ketyl, metal hydride). Reactions were performed in an oven-dried glassware under positive Ar pressure with magnetic stirring. TLC was performed on $0.25 \mathrm{~mm}$ E. Merck silica gel 60 F254 plates and visualized under UV light $(254 \mathrm{~nm})$ or by staining with $p$-anisaldehyde or cerium ammonium molybdate (CAM). Flash chromatography was performed on E. Merch 230-400 mesh silica gel 60. NMR spectra were recorded on Varian Unity 400 instruments at $24^{\circ} \mathrm{C}$. Chemical shifts are expressed in ppm relative to TMS $\left({ }^{1} \mathrm{H}, 0 \mathrm{ppm}\right)$, or $\mathrm{CDCl}_{3}\left({ }^{1} \mathrm{H}\right.$, $\left.7.26 \mathrm{ppm} ;{ }^{13} \mathrm{C}, 77.2 \mathrm{ppm}\right)$; coupling constants are expressed in Hz. High resolution mass spectra electrospray ionization (HRMS-ESI) were obtained on an Agilent technologies 6220 TOF LC/MS spectrometer.

Synthesis of $(Z)$-4-Phenylbut-3-yn-2-one $\boldsymbol{O}$-methyloxime (1a). In an oven-dried roundbottom flask, $\mathrm{NH}_{2} \mathrm{OCH}_{3} \cdot \mathrm{HCl}$ (263 mg, $3.20 \mathrm{mmol}$ ) and NaOAc (258 mg, $3.20 \mathrm{mmol})$ were dissolved in anhydrous EtOH $(10.5 \mathrm{~mL})$ under $\mathrm{Ar}$ atmosphere. 4-Phenyl-3-butyn-2-one (310 $\mu \mathrm{L}, 2.10 \mathrm{mmol})$ was added and the resulting suspension was stirred at $\mathrm{rt}$. After $1.5 \mathrm{~h}, \mathrm{EtOH}$ was evaporated. Then, the residue was diluted with $\mathrm{H}_{2} \mathrm{O}(20 \mathrm{~mL})$ and extracted with EtOAc $(3 \times 20$ $\mathrm{mL})$. The combined organic extracts were dried over anhydrous $\mathrm{MgSO}_{4}$, filtered, and evaporated. Column chromatography (60:1 hexane/EtOAc) afforded 1a (191 mg, 52\%) as a colorless liquid. TLC: $R_{f} 0.14$ (14:1 hexane/EtOAc); IR (KBr film) 2936, 2223, 1599, 1490, 1375, 1313, 1170, 1061, $757,690 \mathrm{~cm}^{-1}$; ${ }^{1} \mathrm{H}-\mathrm{NMR}\left(400 \mathrm{MHz}, \mathrm{CDCl}_{3}\right) \delta 7.54-7.51(\mathrm{~m}$, 2H), 7.40-7.32 (m, 3H), 3.97 (s, 3H), $2.13(\mathrm{~s}, 3 \mathrm{H}) ;{ }^{13} \mathrm{C}-\mathrm{NMR}$ $\left(100 \mathrm{MHz}, \mathrm{CDCl}_{3}\right) \delta 137.7,132.3,129.6,128.6,121.9,99.3$, 81.2, 62.5, 20.8; LRMS (ESI) $m / z$ (rel int): $174\left([\mathrm{M}+\mathrm{H}]^{+}\right.$, 100), $128\left(\left[\mathrm{M}-\mathrm{NOCH}_{3}\right]^{+}, 46\right)$; HRMS (ESI) $\mathrm{m} / z$ calcd for $\mathrm{C}_{11} \mathrm{H}_{11} \mathrm{NO}$ 173.0841, found 173.0840.

Representative Procedure for the Synthesis of 3-Methyl5-phenyl-4-phenylethynylisoxazole (4a). To a stirring solution of $\mathrm{K}_{3} \mathrm{PO}_{4}(170 \mathrm{mg}, 800 \mu \mathrm{mol}), \mathrm{PPh}_{3} \mathrm{AuNTf}_{2}(29.6 \mathrm{mg}$, $40.0 \mu \mathrm{mol}$ ) and Selectfluor (354 mg, $1.00 \mathrm{mmol}$ ) in MeCN $(4 \mathrm{~mL})$ were added $(Z)$-4-phenylbut-3-yn-2-one $O$-methyloxime (1a) (69.3 mg, $400 \mu \mathrm{mol})$, phenylacetylene (3a) $(88.0$ $\mu \mathrm{L}, 800 \mu \mathrm{mol})$ and $\mathrm{H}_{2} \mathrm{O}(72.0 \mu \mathrm{L})$ sequentially under $\mathrm{Ar}$ atmosphere. The resulting suspension was stirred at $\mathrm{rt}$ for 17 h. After the completion of the reaction, the reaction mixture was diluted with $\mathrm{H}_{2} \mathrm{O}(20 \mathrm{~mL})$ and extracted with EtOAc (3 $\times 20 \mathrm{~mL}$ ). The combined organic extracts were dried over anhydrous $\mathrm{MgSO}_{4}$, filtered, and evaporated. Purification by column chromatography (35:1 hexane/EtOAc) afforded 4a
(52 $\mathrm{mg}, 50 \%)$ as a yellow solid, 5 (12.6 $\mathrm{mg}, 20 \%)$ as a yellow solid, 6 (12.9 mg, 18\%) as a yellow liquid, and 7a (43.6 mg, 27\%) as a white solid.

4a: $\mathrm{mp} 67-69{ }^{\circ} \mathrm{C}$ (from EtOAc); TLC: $R_{f} 0.20$ (14:1 hexane/EtOAc); ${ }^{1} \mathrm{H}-\mathrm{NMR}\left(400 \mathrm{MHz}, \mathrm{CDCl}_{3}\right) \delta 8.20-8.17$ (m, 2H), 7.57-7.55 (m, 2H), 7.53-7.47 (m, 3H), 7.40-7.39 (m, 3H), 2.44 (s, 3H); ${ }^{13} \mathrm{C}-\mathrm{NMR}\left(100 \mathrm{MHz}, \mathrm{CDCl}_{3}\right) \delta 168.4$, $162.0,131.7,130.8,129.1,129.0,128.7,127.5,126.4$, 122.9, 99.3, 96.3, 78.9, 10.8; LRMS (ESI) $m / z$ (rel int): 260 $\left([\mathrm{M}+\mathrm{H}]^{+}, 84\right), 219\left(\left[\mathrm{M}-\mathrm{CH}_{2} \mathrm{CN}\right]^{+}, 100\right) ; \operatorname{HRMS}(\mathrm{ESI}) \mathrm{m} / z$ calcd for $\mathrm{C}_{18} \mathrm{H}_{13} \mathrm{NO} 259.0997$, found 259.0997.

5: $\mathrm{mp} 76-78^{\circ} \mathrm{C}$ (from EtOAc) (Lit. ${ }^{25} 65-68{ }^{\circ} \mathrm{C}$ ); TLC: $R_{f}$ 0.13 (14:1 hexane/EtOAc); ${ }^{1} \mathrm{H}-\mathrm{NMR}\left(400 \mathrm{MHz}, \mathrm{CDCl}_{3}\right) \delta$ $7.77-7.75(\mathrm{~m}, 2 \mathrm{H}), 7.48-7.42(\mathrm{~m}, 3 \mathrm{H}), 6.36(\mathrm{~s}, 1 \mathrm{H}), 2.36(\mathrm{~s}$, $3 \mathrm{H}) ;{ }^{13} \mathrm{C}-\mathrm{NMR}\left(100 \mathrm{MHz}, \mathrm{CDCl}_{3}\right) \delta 169.9,160.6,130.2$, 129.1, 127.8, 126.0, 100.4, 11.7; LRMS (ESI) $m / z$ (rel int): $160\left([\mathrm{M}+\mathrm{H}]^{+}, 100\right)$; HRMS (ESI) $m / z$ calcd for $\mathrm{C}_{10} \mathrm{H}_{9} \mathrm{NO}$ 159.0684, found 159.0682 .

6: TLC: $R_{f} 0.26$ (14:1 hexane/EtOAc); ${ }^{1} \mathrm{H}-\mathrm{NMR}(400$ $\left.\mathrm{MHz}, \mathrm{CDCl}_{3}\right) \delta 7.82-7.79(\mathrm{~m}, 2 \mathrm{H}), 7.51-7.47(\mathrm{~m}, 2 \mathrm{H}), 7.45$ $-7.41(\mathrm{~m}, 1 \mathrm{H}), 2.37(\mathrm{~s}, 3 \mathrm{H}) ;{ }^{13} \mathrm{C}-\mathrm{NMR}\left(100 \mathrm{MHz}, \mathrm{CDCl}_{3}\right) \delta$ $152.3\left(\mathrm{~d}, J_{\mathrm{C}-\mathrm{F}}=9.4 \mathrm{~Hz}\right), 152.1\left(\mathrm{~d}, J_{\mathrm{C}-\mathrm{F}}=7.1 \mathrm{~Hz}\right), 142.5(\mathrm{~d}$, $\left.J_{\mathrm{C}-\mathrm{F}}=253.9 \mathrm{~Hz}\right), 130.1,129.2,126.2\left(\mathrm{~d}, J_{\mathrm{C}-\mathrm{F}}=5.0 \mathrm{~Hz}\right), 125.4$ $\left(\mathrm{d}, J_{\mathrm{C}-\mathrm{F}}=3.4 \mathrm{~Hz}\right), 8.9\left(\mathrm{~d}, J_{\mathrm{C}-\mathrm{F}}=2.9 \mathrm{~Hz}\right) ;{ }^{1} \mathrm{~F}-\mathrm{NMR}(376 \mathrm{MHz}$, $\left.\mathrm{CDCl}_{3}\right) \delta-180.5(\mathrm{~s}, 1 \mathrm{~F}) ;$ LRMS (ESI) $m / z$ (rel int): 178 ([M $\left.+\mathrm{H}]^{+}, 2\right), 105\left(\left[\mathrm{M}-\mathrm{NCCH}_{3} \mathrm{CF}\right]^{+}, 100\right)$. HRMS (ESI) $\mathrm{m} / z$ calcd for $\mathrm{C}_{10} \mathrm{H}_{8} \mathrm{FNO} 177.0590$, found 177.0590.

7a: mp $85-87{ }^{\circ} \mathrm{C}$ (from EtOAc) (Lit., ${ }^{26} 82-85{ }^{\circ} \mathrm{C}$ ); TLC: $R_{f} 0.44$ (14:1 hexane/EtOAc); ${ }^{1} \mathrm{H}-\mathrm{NMR}\left(400 \mathrm{MHz}, \mathrm{CDCl}_{3}\right)$ $\delta$ 7.55-7.52 (m, 4H), 7.40-7.32 (m, 6H); ${ }^{13} \mathrm{C}-\mathrm{NMR}(100$ $\left.\mathrm{MHz}, \mathrm{CDCl}_{3}\right) \delta 132.7,129.4,128.6,122.0,81.8,74.1$; LRMS (ESI) $m / z$ (rel int): $405\left([2 \mathrm{M}+\mathrm{H}]^{+}, 8\right) .203\left([\mathrm{M}+\mathrm{H}]^{+}, 28\right)$; HRMS (ESI) $m / z$ calcd for $\mathrm{C}_{16} \mathrm{H}_{10} 202.0783$, found 202.0784 .

Synthesis of 4-(2-Chlorophenylethynyl)-3-methyl-5phenylisoxazole (4b). 1-Chloro-2-ethynylbenzene (3b) was used. The suspension was stirred for $25 \mathrm{~h}$ at rt. Column chromatography (50:1 hexane/EtOAc) afforded 4b (55.0 $\mathrm{mg}, 47 \%)$ as a yellow solid, $5(13.8 \mathrm{mg}, 22 \%)$ as a yellow solid, 6 (16.5 mg, 23\%) as a yellow liquid, and $7 \mathbf{b}$ (47 mg, $22 \%$ ) as a white solid.

4b: $\mathrm{mp} 71-73{ }^{\circ} \mathrm{C}$ (from EtOAc); TLC: $R_{f} 0.15$ (14:1 hexane/EtOAc); ${ }^{1} \mathrm{H}-\mathrm{NMR}\left(400 \mathrm{MHz}, \mathrm{CDCl}_{3}\right) \delta 8.27-8.25$ (m, 2H), 7.59-7.57 (m, 1H), 7.53-7.46 (m, 4H), 7.34-7.26 $(\mathrm{m}, 2 \mathrm{H}), 2.47(\mathrm{~s}, 3 \mathrm{H}) ;{ }^{13} \mathrm{C}-\mathrm{NMR}\left(100 \mathrm{MHz}, \mathrm{CDCl}_{3}\right) \delta 168.8$, $162.1,135.9,133.3,130.9,129.9,129.7,129.0,127.3$, 126.8, 126.6, 123.0, 99.1, 93.2, 84.1, 10.8; LRMS (ESI) $\mathrm{m} / \mathrm{z}$ (rel int): $294\left([\mathrm{M}+\mathrm{H}]^{+}, 100\right), 253\left(\left[\mathrm{M}-\mathrm{CH}_{2} \mathrm{CN}\right]^{+}, 53\right)$; HRMS (ESI) $m / z$ calcd for $\mathrm{C}_{18} \mathrm{H}_{12} \mathrm{CINO} 293.0607$, found 293.0608 .

7b: mp 142-144 ${ }^{\circ} \mathrm{C}$ (from EtOAc) (Lit., ${ }^{27} 138-140{ }^{\circ} \mathrm{C}$ ); TLC: $R_{f} 0.39$ (14:1 hexane/EtOAc); ${ }^{1} \mathrm{H}-\mathrm{NMR}(400 \mathrm{MHz}$, $\left.\mathrm{CDCl}_{3}\right) \delta 7.58(\mathrm{ddd}, 2 \mathrm{H}, J=8.0,1.6,0.4 \mathrm{~Hz}), 7.42(\mathrm{ddd}, 2 \mathrm{H}$, $J=8.0,1.6,0.4 \mathrm{~Hz}), 7.31(\mathrm{td}, 2 \mathrm{H}, J=8.0,1.6 \mathrm{~Hz}), 7.24(\mathrm{td}$, $2 \mathrm{H}, J=8.0,1.6 \mathrm{~Hz}) ;{ }^{13} \mathrm{C}-\mathrm{NMR}\left(100 \mathrm{MHz}, \mathrm{CDCl}_{3}\right) \delta 137.2$, 134.6, 130.5, 129.7, 126.8, 122.0, 79.6, 78.6; LRMS (ESI) $\mathrm{m} / z$ (rel int): $271\left([\mathrm{M}+\mathrm{H}]^{+}, 100\right)$; HRMS (ESI) $m / z$ calcd for $\mathrm{C}_{16} \mathrm{H}_{8} \mathrm{Cl}_{2} 270.0003$, found 270.0004 . 
Synthesis of 4-(3-Chlorophenylethynyl)-3-methyl-5phenylisoxazole (4c). 1-Chloro-3-ethynylbenzene (3c) was used. The suspension was stirred for $17 \mathrm{~h}$ at rt. Column chromatography (40:1 hexane/EtOAc) afforded $\mathbf{4 c}(40.0 \mathrm{mg}$, $34 \%)$ as a yellow solid, $5(19.0 \mathrm{mg}, 30 \%)$ as a yellow solid, $6(15.0 \mathrm{mg}, 21 \%)$ as a yellow liquid, and $7 \mathrm{c}(23.0 \mathrm{mg}, 11 \%)$ as a white solid.

4c: $\mathrm{mp}$ 81-83 ${ }^{\circ} \mathrm{C}$ (from EtOAc); TLC: $R_{f} 0.25$ (14:1 hexane/EtOAc); ${ }^{1} \mathrm{H}-\mathrm{NMR}\left(400 \mathrm{MHz}, \mathrm{CDCl}_{3}\right) \delta 8.16-8.14$ $(\mathrm{m}, 2 \mathrm{H}), 7.54-7.48(\mathrm{~m}, 4 \mathrm{H}), 7.34(\mathrm{dt}, 1 \mathrm{H}, J=7.6,2.0 \mathrm{~Hz})$, 7.38-7.30 (m, 2H), $2.43(\mathrm{~s}, 3 \mathrm{H}) ;{ }^{13} \mathrm{C}-\mathrm{NMR}(100 \mathrm{MHz}$, $\left.\mathrm{CDCl}_{3}\right) \delta 168.9,162.0,134.6,131.5,131.0,130.0,129.8$, 129.2, 129.1, 127.4, 126.5, 124.6, 98.8, 94.7, 80.1, 10.8; LRMS (ESI) $m / z$ (rel int): $294\left([\mathrm{M}+\mathrm{H}]^{+}, 100\right), 253$ ([M $\left.\mathrm{CH}_{2} \mathrm{CN}\right]^{+}$, 53); HRMS (ESI) $m / z$ calcd for $\mathrm{C}_{18} \mathrm{H}_{12} \mathrm{CINO}$ 293.0607, found 293.0610.

7c: $\mathrm{mp} 78-80{ }^{\circ} \mathrm{C}$ (from EtOAc) (Lit., ${ }^{26} 73{ }^{\circ} \mathrm{C}$ ); TLC: $R_{f}$ 0.5 (14:1 hexane/EtOAc); IR (KBr film) 3066, 1583, 1557, 1470, 1455, 1401, 1261, 1164, 1093, 1076, 907, 890, 850, 793, 776, 675, $522 \mathrm{~cm}^{-1} ;{ }^{1} \mathrm{H}-\mathrm{NMR}\left(400 \mathrm{MHz}, \mathrm{CDCl}_{3}\right) \delta$ $7.51(\mathrm{~m}, 2 \mathrm{H}), 7.41(\mathrm{~d}, 2 \mathrm{H}, J=8.0 \mathrm{~Hz}), 7.38-7.32(\mathrm{~m}, 2 \mathrm{H})$, $7.28(\mathrm{t}, 2 \mathrm{H}, J=8.0 \mathrm{~Hz}) ;{ }^{13} \mathrm{C}-\mathrm{NMR}\left(100 \mathrm{MHz}, \mathrm{CDCl}_{3}\right) \delta$ 134.6, 132.5, 130.9, 129.9, 129.4, 123.5, 80.8, 74.9.

Synthesis of 4-(3,5-Difluorophenylethynyl)-3-methyl-5phenylisoxazole (4d). $\mathrm{K}_{3} \mathrm{PO}_{4}(85.0 \mathrm{mg}, 400 \mu \mathrm{mol}), \mathrm{PPh}_{3} \mathrm{AuNTf}_{2}$ (14.8 mg, $20.0 \mu \mathrm{mol})$, Selectfluor $(177 \mathrm{mg}, 500 \mu \mathrm{mol}), \mathrm{MeCN}$ (2 mL), (Z)-4-phenylbut-3-yn-2-one $O$-methyloxime (1a) (34.6 mg, $200 \mu \mathrm{mol}$ ), 1-ethynyl-3,5-difluorobenzene (3d) $(48.0 \mu \mathrm{L}, 400 \mu \mathrm{mol})$ and $\mathrm{H}_{2} \mathrm{O}(36.0 \mu \mathrm{L})$ were used. The suspension was stirred for $22 \mathrm{~h}$ at rt. Column chromatography (40:1 hexane/EtOAc) afforded $\mathbf{4 d}(26.8 \mathrm{mg}, 45 \%)$ as a yellow solid, $\mathbf{5}(6.8 \mathrm{mg}, 21 \%)$ as a yellow solid, $\mathbf{6}(9.2 \mathrm{mg}$, $26 \%)$ as a yellow liquid, and $7 \mathbf{d}(17.0 \mathrm{mg}, 16 \%)$ as a white solid.

4d: $m p$ 99-101 ${ }^{\circ} \mathrm{C}$ (from EtOAc); TLC: $R_{f} 0.24$ (14:1 hexane/EtOAc); ${ }^{1} \mathrm{H}-\mathrm{NMR}\left(400 \mathrm{MHz}, \mathrm{CDCl}_{3}\right) \delta 8.14-8.12$ $(\mathrm{m}, 2 \mathrm{H}), 7.54-7.48(\mathrm{~m}, 3 \mathrm{H}), 7.07-7.04(\mathrm{~m}, 2 \mathrm{H}), 6.86(\mathrm{tt}$, $1 \mathrm{H}, J=8.8,2.0 \mathrm{~Hz}), 2.43(\mathrm{~s}, 3 \mathrm{H}) ;{ }^{13} \mathrm{C}-\mathrm{NMR}(100 \mathrm{MHz}$, $\left.\mathrm{CDCl}_{3}\right) \delta 169.3,163.0\left(\mathrm{dd}, J_{\mathrm{C}-\mathrm{F}}=243.5,11.7 \mathrm{~Hz}\right), 132.3$, $131.1,129.6,129.2,128.6,127.3,126.5,114.7\left(\mathrm{dd}, J_{\mathrm{C}-\mathrm{F}}=\right.$ 19.0, 7.2 Hz), 105.1 (t, $\left.J_{\mathrm{C}-\mathrm{F}}=25.6 \mathrm{~Hz}\right), 81.0,62.5,20.8$; LRMS (ESI) $m / z$ (rel int): $296\left([\mathrm{M}+\mathrm{H}]^{+}, 100\right), 255$ ([M $\left.\mathrm{CH}_{2} \mathrm{CN}\right]^{+}$, 35); HRMS (ESI) $m / z$ calcd for $\mathrm{C}_{18} \mathrm{H}_{11} \mathrm{~F}_{2} \mathrm{NO}$ 295.0809, found 295.0809 .

7d: $\mathrm{mp} 87-89{ }^{\circ} \mathrm{C}$ (from EtOAc); TLC: $R_{f} 0.62$ (14:1 hexane/EtOAc); IR (KBr film) 3100, 1610, 1583, 1456, 1402, 1094, 1077, 960, 890, 794, $681 \mathrm{~cm}^{-1}$; ${ }^{1} \mathrm{H}-\mathrm{NMR}(400$ $\left.\mathrm{MHz} \mathrm{CDCl}_{3}\right) \delta$ 7.07-7.02 (m, 4H), 6.90-6.84 (tt, 2H, $J=$ 8.8, $2.0 \mathrm{~Hz}) ;{ }^{13} \mathrm{C}-\mathrm{NMR}\left(100 \mathrm{MHz}, \mathrm{CDCl}_{3}\right) \delta 162.9\left(\mathrm{dd}, J_{\mathrm{C}-\mathrm{F}}\right.$ $=250,13.5 \mathrm{~Hz}), 124.1\left(\mathrm{t}, J_{\mathrm{C}-\mathrm{F}}=11.9 \mathrm{~Hz}\right), 115.8\left(\mathrm{dd}, J_{\mathrm{C}-\mathrm{F}}=\right.$ $19.1,8.0 \mathrm{~Hz}), 106.2\left(\mathrm{t}, J_{\mathrm{C}-\mathrm{F}}=25.4 \mathrm{~Hz}\right), 80.2\left(\mathrm{~d}, J_{\mathrm{C}-\mathrm{F}}=4.0\right.$ $\mathrm{Hz}), 75.2 ;{ }^{19} \mathrm{~F}-\mathrm{NMR}\left(376 \mathrm{MHz}, \mathrm{CDCl}_{3}\right) \delta-108.7(\mathrm{t}, 4 \mathrm{~F}, J=$ $8.3 \mathrm{~Hz})$.

Synthesis of 4-(3-Fluorophenylethynyl)-3-methyl-5-phenylisoxazole (4e). $\mathrm{K}_{3} \mathrm{PO}_{4}(85.0 \mathrm{mg}, 400 \mu \mathrm{mol}), \mathrm{PPh}_{3} \mathrm{AuNTf}_{2}$ (14.8 mg, $20.0 \mu \mathrm{mol})$, Selectfluor $(177 \mathrm{mg}, 500 \mu \mathrm{mol}), \mathrm{MeCN}$ (2 mL), (Z)-4-phenylbut-3-yn-2-one $O$-methyloxime (1a)
(34.6 mg, $200 \mu \mathrm{mol}$ ), 1-ethynyl-3-fluorobenzene (3e) (46.0 $\mu \mathrm{L}, 400 \mu \mathrm{mol})$ and $\mathrm{H}_{2} \mathrm{O}(36.0 \mu \mathrm{L})$ were used. The suspension was stirred for $20 \mathrm{~h}$ at $60{ }^{\circ} \mathrm{C}$. Column chromatography (40:1 hexane/EtOAc) afforded $4 \mathrm{e}(23.0 \mathrm{mg}, 41 \%)$ as a yellow solid, $5(8.7 \mathrm{mg}, 27 \%)$ as a yellow solid, $6(9.1 \mathrm{mg}$, $26 \%$ ) as a yellow liquid, and 7 e (18.2 $\mathrm{mg}, 19 \%)$ as a white solid.

4e: $\mathrm{mp} 79-81{ }^{\circ} \mathrm{C}$ (from EtOAc); TLC: $R_{f} 0.22$ (14:1 hexane/EtOAc); ${ }^{1} \mathrm{H}-\mathrm{NMR}\left(400 \mathrm{MHz}, \mathrm{CDCl}_{3}\right) \delta 8.17-8.14$ (m, 2H), 7.54-7.47 (m, 3H), 7.39-7.32 (m, 2H), 7.27-7.23 $(\mathrm{m}, 1 \mathrm{H}), 7.12-7.07(\mathrm{~m}, 1 \mathrm{H}), 2.44(\mathrm{~s}, 3 \mathrm{H}) ;{ }^{13} \mathrm{C}-\mathrm{NMR}(100$ $\left.\mathrm{MHz}, \mathrm{CDCl}_{3}\right) \delta 168.9,162.7\left(\mathrm{~d}, J_{\mathrm{C}-\mathrm{F}}=246.0 \mathrm{~Hz}\right), 162.0$, $131.0,130.4\left(\mathrm{~d}, J_{\mathrm{C}-\mathrm{F}}=8.7 \mathrm{~Hz}\right), 129.1,127.6\left(\mathrm{~d}, J_{\mathrm{C}-\mathrm{F}}=2.9\right.$ $\mathrm{Hz}), 127.4,126.5,124.7\left(\mathrm{~d}, J_{\mathrm{C}-\mathrm{F}}=9.4 \mathrm{~Hz}\right), 118.5\left(\mathrm{~d}, J_{\mathrm{C}-\mathrm{F}}=\right.$ $22.9 \mathrm{~Hz}), 116.4\left(\mathrm{~d}, J_{\mathrm{C}-\mathrm{F}}=21.6 \mathrm{~Hz}\right), 98.9,94.9,79.9,10.8$. LRMS (ESI) $m / z$ (rel int): $278\left([\mathrm{M}+\mathrm{H}]^{+}, 100\right), 237$ ([M $\mathrm{CH}_{2} \mathrm{CN}^{+}$, 62); HRMS (ESI) $m / z$ calcd for $\mathrm{C}_{18} \mathrm{H}_{12} \mathrm{FNO}$ 277.0903, found 277.0903.

7e: $\mathrm{mp} 122-124{ }^{\circ} \mathrm{C}$ (from EtOAc) (Lit., ${ }^{28} 122-123{ }^{\circ} \mathrm{C}$ ); TLC: $R_{f} 0.40$ (14:1 hexane/EtOAc); IR (KBr film) 3030, 1604, 1583, 1482, 1461, 1428, 1265, 1130, 1078, 933, 868, $782 \mathrm{~cm}^{-1} ;{ }^{1} \mathrm{H}-\mathrm{NMR}\left(400 \mathrm{MHz}, \mathrm{CDCl}_{3}\right) \delta 7.33-7.30(\mathrm{~m}$, 4H), 7.21-7.20 (m, 2H), 7.12-7.07 (m, 2H); ${ }^{13} \mathrm{C}-\mathrm{NMR}(100$ $\left.\mathrm{MHz} \mathrm{CDCl}_{3}\right) \delta 162.5\left(\mathrm{~d}, J_{\mathrm{C}-\mathrm{F}}=240.8 \mathrm{~Hz}\right), 130.4\left(\mathrm{~d}, J_{\mathrm{C}-\mathrm{F}}=\right.$ $8.7 \mathrm{~Hz}), 128.7\left(\mathrm{~d}, J_{\mathrm{C}-\mathrm{F}}=3.6 \mathrm{~Hz}\right), 123.6\left(\mathrm{~d}, J_{\mathrm{C}-\mathrm{F}}=9.4 \mathrm{~Hz}\right)$, $119.5\left(\mathrm{~d}, J_{\mathrm{C}-\mathrm{F}}=23.0 \mathrm{~Hz}\right), 117.1\left(\mathrm{~d}, J_{\mathrm{C}-\mathrm{F}}=21.6 \mathrm{~Hz}\right), 80.8(\mathrm{~d}$, $\left.J_{\mathrm{C}-\mathrm{F}}=2.8 \mathrm{~Hz}\right), 74.6 ;{ }^{19} \mathrm{~F}-\mathrm{NMR}\left(376 \mathrm{MHz}, \mathrm{CDCl}_{3}\right) \delta-112.2-$ $-112.3(\mathrm{~m}, 2 \mathrm{~F})$.

Synthesis of 4-(2,4-Difluorophenylethynyl)-3-methyl-5phenylisoxazole (4f). $\mathrm{K}_{3} \mathrm{PO}_{4}(85.0 \mathrm{mg}, 400 \mu \mathrm{mol}), \mathrm{PPh}_{3}-$ AuNTf $_{2}(14.8 \mathrm{mg}, 20 \mu \mathrm{mol})$, Selectfluor $(177 \mathrm{mg}, 500 \mu \mathrm{mol})$, MeCN (2 mL), (Z)-4-phenylbut-3-yn-2-one $O$-methyloxime (1a) $(34.6 \mathrm{mg}, 200 \mu \mathrm{mol})$, 1-ethynyl-2,4-difluorobenzene (3f) $(55.0 \mu \mathrm{L}, 400 \mu \mathrm{mol})$ and $\mathrm{H}_{2} \mathrm{O}(36.0 \mu \mathrm{L})$ were used. The suspension was stirred for $24 \mathrm{~h}$ at $60{ }^{\circ} \mathrm{C}$. Column chromatography (40:1 hexane/EtOAc) afforded $\mathbf{4 f}(17.0 \mathrm{mg}, 30 \%)$ as a yellow solid, $5(2.7 \mathrm{mg}, 8 \%)$ as a yellow solid, $6(8.4 \mathrm{mg}$, $24 \%)$ as a yellow liquid, and $7 \mathbf{f}(9.1 \mathrm{mg}, 8 \%)$ as a yellow solid.

4f: $\mathrm{mp}$ 87-89 ${ }^{\circ} \mathrm{C}$ (from EtOAc); TLC: $R_{f} 0.36$ (14:1 hexane/EtOAc); ${ }^{1} \mathrm{H}-\mathrm{NMR}\left(400 \mathrm{MHz}, \mathrm{CDCl}_{3}\right) \delta 8.21-8.18$ $(\mathrm{m}, 2 \mathrm{H}), 7.54-7.48(\mathrm{~m}, 4 \mathrm{H}), 6.95-6.90(\mathrm{~m}, 2 \mathrm{H}), 2.44(\mathrm{~s}$, $3 \mathrm{H}) ;{ }^{13} \mathrm{C}-\mathrm{NMR}\left(100 \mathrm{MHz}, \mathrm{CDCl}_{3}\right) \delta 168.9,164.5\left(\mathrm{~d}, J_{\mathrm{C}-\mathrm{F}}=\right.$ $11.2 \mathrm{~Hz}), 163.3\left(\mathrm{dd}, J_{\mathrm{C}-\mathrm{F}}=240.0,19.0 \mathrm{~Hz}\right), 161.9,134.1(\mathrm{dd}$, $\left.J_{\mathrm{C}-\mathrm{F}}=9.9,2.8 \mathrm{~Hz}\right), 131.0,129.1,127.3,126.5,118.8,112.0$ $\left(\mathrm{dd}, J_{\mathrm{C}-\mathrm{F}}=21.8,3.5 \mathrm{~Hz}\right), 104.7\left(\mathrm{t}, J_{\mathrm{C}-\mathrm{F}}=25.0 \mathrm{~Hz}\right), 98.9,88.7$, 83.9, 10.8; LRMS (ESI) $m / z$ (rel int): $296\left([\mathrm{M}+\mathrm{H}]^{+}, 100\right)$, $255\left(\left[\mathrm{M}-\mathrm{CH}_{2} \mathrm{CN}\right]^{+}, 63\right)$; HRMS (ESI) $\mathrm{m} / z$ calcd for $\mathrm{C}_{18} \mathrm{H}_{11} \mathrm{~F}_{2} \mathrm{NO} 295.0809$, found 295.0811.

7f: $\mathrm{mp} 168-170{ }^{\circ} \mathrm{C}$ (from EtOAc) (Lit., ${ }^{29} 187-188^{\circ} \mathrm{C}$ ); TLC: $R_{f} 0.48$ (14:1 hexane/EtOAc); ${ }^{1} \mathrm{H}-\mathrm{NMR}(400 \mathrm{MHz}$, $\left.\mathrm{CDCl}_{3}\right) \delta 7.53-7.48(\mathrm{~m}, 2 \mathrm{H}), 6.91-6.84(\mathrm{~m}, 4 \mathrm{H}) ;{ }^{13} \mathrm{C}-\mathrm{NMR}$ $\left(100 \mathrm{MHz}, \mathrm{CDCl}_{3}\right) \delta 164.6\left(\mathrm{dd}, J_{\mathrm{C}-\mathrm{F}}=205.0,11.0 \mathrm{~Hz}\right), 163.7$ $\left(\mathrm{dd}, J_{\mathrm{C}-\mathrm{F}}=202.0,9.1 \mathrm{~Hz}\right), 135.5\left(\mathrm{dd}, J_{\mathrm{C}-\mathrm{F}}=7.9,1.8 \mathrm{~Hz}\right)$, $112.2\left(\mathrm{dd}, J_{\mathrm{C}-\mathrm{F}}=17.6,3.0 \mathrm{~Hz}\right), 107.0\left(\mathrm{dd}, J_{\mathrm{C}-\mathrm{F}}=12.8,3.7\right.$ $\mathrm{Hz}), 104.8\left(\mathrm{t}, J_{\mathrm{C}-\mathrm{F}}=20.6 \mathrm{~Hz}\right), 78.3,75.1$; LRMS (ESI) $\mathrm{m} / \mathrm{z}$ (rel int): $275\left([\mathrm{M}+\mathrm{H}]^{+}, 100\right)$; HRMS (ESI) $\mathrm{m} / z$ calcd for 
$\mathrm{C}_{16} \mathrm{H}_{6} \mathrm{~F}_{4} 274.0406$, found 274.0404.

Synthesis of 4-(2-Fluorophenylethynyl)-3-methyl-5-phenylisoxazole (4g). 1-Ethynyl-2-fluorobenzene (3g) was used. The suspension was stirred for $20 \mathrm{~h}$ at rt. Column chromatography (50:1 hexane/EtOAc) afforded $\mathbf{4 g}(40.3 \mathrm{mg}, 36 \%)$ as a yellow solid, 5 (11.9 mg, 19\%) as a yellow solid, 6 (3.7 $\mathrm{mg}, 5 \%)$ as a yellow liquid, and $7 \mathrm{~g}(49.9 \mathrm{mg}, 26 \%)$ as a yellow solid.

4g: $\mathrm{mp} 68-70{ }^{\circ} \mathrm{C}$ (from EtOAc); TLC: $R_{f} 0.33$ (14:1 hexane/EtOAc); ${ }^{1} \mathrm{H}-\mathrm{NMR}\left(400 \mathrm{MHz}, \mathrm{CDCl}_{3}\right) \delta 8.23-8.20$ $(\mathrm{m}, 2 \mathrm{H}), 7.55-7.46(\mathrm{~m}, 4 \mathrm{H}), 7.40-7.34(\mathrm{~m}, 1 \mathrm{H}), 7.17(\mathrm{td}$, $1 \mathrm{H}, J=7.6,1.2 \mathrm{~Hz}), 7.16-7.13(\mathrm{~m}, 1 \mathrm{H}), 2.45(\mathrm{~s}, 3 \mathrm{H}) ;{ }^{13} \mathrm{C}-$ NMR $\left(100 \mathrm{MHz}, \mathrm{CDCl}_{3}\right) \delta 168.8,162.9\left(\mathrm{~d}, J_{\mathrm{C}-\mathrm{F}}=250.3 \mathrm{~Hz}\right)$, $162.0,133.2,130.9,130.7\left(\mathrm{~d}, J_{\mathrm{C}-\mathrm{F}}=7.6 \mathrm{~Hz}\right), 129.1,127.4$, $126.5,124.3\left(\mathrm{~d}, J_{\mathrm{C}-\mathrm{F}}=3.1 \mathrm{~Hz}\right), 115.9\left(\mathrm{~d}, J_{\mathrm{C}-\mathrm{F}}=20.6 \mathrm{~Hz}\right)$, $111.7\left(\mathrm{~d}, J_{\mathrm{C}-\mathrm{F}}=16.0 \mathrm{~Hz}\right), 99.1,89.9,84.1\left(\mathrm{~d}, J_{\mathrm{C}-\mathrm{F}}=3.8 \mathrm{~Hz}\right)$, 10.8; LRMS (ESI) $\mathrm{m} / z$ (rel int): 278 ([M + H] $\left.]^{+}, 100\right), 236$ $\left(\left[\mathrm{M}-\mathrm{CH}_{3} \mathrm{CN}\right]^{+}, 80\right)$; HRMS (ESI) $m / z$ calcd for $\mathrm{C}_{18} \mathrm{H}_{12} \mathrm{FNO}$ 277.0903 , found 277.0904 .

7g: mp 144-146 ${ }^{\circ} \mathrm{C}$ (from EtOAc); TLC: $R_{f} 0.42$ (14:1 hexane/EtOAc); ${ }^{1} \mathrm{H}-\mathrm{NMR}\left(400 \mathrm{MHz}, \mathrm{CDCl}_{3}\right) \delta 7.52(\mathrm{td}, 2 \mathrm{H}$, $J=7.6,2.0 \mathrm{~Hz}), 7.39-7.34$ (m, 2H), 7.13 (td, 2H, $J=7.6,1.2$ $\mathrm{Hz}), 7.11-7.08(\mathrm{~m}, 2 \mathrm{H}) ;{ }^{13} \mathrm{C}-\mathrm{NMR}\left(100 \mathrm{MHz} \mathrm{CDCl}_{3}\right) \delta$ $164.0\left(\mathrm{~d}, J_{\mathrm{C}-\mathrm{F}}=251.8 \mathrm{~Hz}\right), 134.5,131.3\left(\mathrm{~d}, J_{\mathrm{C}-\mathrm{F}}=7.6 \mathrm{~Hz}\right)$, $124.3\left(\mathrm{~d}, J_{\mathrm{C}-\mathrm{F}}=3.1 \mathrm{~Hz}\right), 115.9\left(\mathrm{~d}, J_{\mathrm{C}-\mathrm{F}}=20.6 \mathrm{~Hz}\right), 110.7(\mathrm{~d}$, $\left.J_{\mathrm{C}-\mathrm{F}}=15.3 \mathrm{~Hz}\right), 78.6,76.1$; LRMS (ESI) $\mathrm{m} / z$ (rel int): 477 $\left([2 \mathrm{M}+\mathrm{H}]^{+}, 6\right), 239\left([\mathrm{M}+\mathrm{H}]^{+}, 100\right)$. HRMS (ESI) $m / z$ calcd for $\mathrm{C}_{16} \mathrm{H}_{8} \mathrm{~F}_{2} 238.0594$, found 238.0591 .

Synthesis of 4-(3,5-Bis(trifluoromethyl)phenylethynyl)3-methyl-5-phenylisoxazole (4h). 1-Ethynyl-3,5-bis(trifluoromethyl)benzene (3h) was used. The suspension was stirred for $18 \mathrm{~h}$ at rt. Column chromatography (50:1 hexane/EtOAc) afforded $\mathbf{4 h}(48.2 \mathrm{mg}, 30 \%)$ as a white solid, 5 (12.4 mg, $19 \%)$ as a yellow solid, $6(10 \mathrm{mg}, 14 \%)$ as a yellow liquid, and $7 \mathrm{~h}(36.2 \mathrm{mg}, 10 \%)$ as a brown solid .

4h: $\mathrm{mp} 124-126^{\circ} \mathrm{C}$ (from EtOAc); TLC: $R_{f} 0.19$ (14:1 hexane/EtOAc); ${ }^{1} \mathrm{H}-\mathrm{NMR}\left(400 \mathrm{MHz}, \mathrm{CDCl}_{3}\right) \delta$ 8.14-8.11 $(\mathrm{m}, 2 \mathrm{H}), 7.95(\mathrm{~s}, 2 \mathrm{H}), 7.87(\mathrm{~s}, 1 \mathrm{H}), 7.57-7.48(\mathrm{~m}, 3 \mathrm{H}), 2.46$ $(\mathrm{s}, 3 \mathrm{H}) ;{ }^{13} \mathrm{C}-\mathrm{NMR}\left(100 \mathrm{MHz}, \mathrm{CDCl}_{3}\right) \delta 169.8,161.9,132.3$ $\left(\mathrm{q}, J_{\mathrm{C}-\mathrm{F}}=33.0 \mathrm{~Hz}\right), 131.5\left(\mathrm{~d}, J_{\mathrm{C}-\mathrm{F}}=3.5 \mathrm{~Hz}\right), 131.3,129.3$, 127.1, 126.6, 125.3, $123.1\left(\mathrm{q}, J_{\mathrm{C}-\mathrm{F}}=270.0 \mathrm{~Hz}\right), 122.3(\mathrm{~m})$, 98.1, 92.9, 82.6, 10.8; LRMS (ESI) $m / z$ (rel int): 396 ([M + $\mathrm{H}]^{+}, 91$ ); HRMS (ESI) $m / z$ calcd for $\mathrm{C}_{20} \mathrm{H}_{11} \mathrm{~F}_{6} \mathrm{NO} 395.0745$, found 395.0746 .

7h: mp $146-148{ }^{\circ} \mathrm{C}$ (from EtOAc) (Lit., ${ }^{30} 158.7-161.0$ $\left.{ }^{\circ} \mathrm{C}\right)$; TLC: $R_{f} 0.55$ (14:1 hexane/EtOAc); IR (KBr film) 3095, 2219, 1615, 1463, 1281, 1262, 1167, 1107, 1022, 899, 801, 699, $684 \mathrm{~cm}^{-1} ;{ }^{1} \mathrm{H}-\mathrm{NMR}\left(400 \mathrm{MHz}, \mathrm{CDCl}_{3}\right) \delta 7.55-7.52$ $(\mathrm{m}, 4 \mathrm{H}), 7.40-7.32(\mathrm{~m}, 2 \mathrm{H}) ;{ }^{13} \mathrm{C}-\mathrm{NMR}\left(100 \mathrm{MHz}, \mathrm{CDCl}_{3}\right) \delta$ $132.5\left(\mathrm{q}, J_{\mathrm{C}-\mathrm{F}}=33.7 \mathrm{~Hz}\right), 132.6\left(\mathrm{~d}, J_{\mathrm{C}-\mathrm{F}}=2.9 \mathrm{~Hz}\right), 123.8$, $123.3\left(\mathrm{t}, J_{\mathrm{C}-\mathrm{F}}=3.6 \mathrm{~Hz}\right), 121.6,118.8,79.9,76.3 ;{ }^{19} \mathrm{~F}-\mathrm{NMR}$ $\left(376 \mathrm{MHz} \mathrm{CDCl}_{3}\right): \delta-63.3(\mathrm{~s}, 12 \mathrm{~F})$.

Synthesis of 4-(2-(3-Methyl-5-phenylisoxazol-4-yl)ethynyl)benzonitrile (4i). 4-Ethynylbenzonitrile (3i) was used. The suspension was stirred for $40 \mathrm{~h}$ at rt. Column chromatography (40:1 hexane/EtOAc) afforded $4 \mathbf{i}(36.7 \mathrm{mg}, 32 \%)$ as a yellow solid, 5 (10 mg, 16\%) as a yellow solid, 6 (15.4 mg,
$22 \%$ ) as a yellow liquid.

4i: $\mathrm{mp} 138-140{ }^{\circ} \mathrm{C}$ (from EtOAc); TLC: $R_{f} 0.16$ (14:1 hexane/EtOAc); ${ }^{1} \mathrm{H}-\mathrm{NMR}\left(400 \mathrm{MHz}, \mathrm{CDCl}_{3}\right) \delta 8.15-8.13$ (m, 2H), 7.70-7.67 (m, 2H), 7.64-7.61 (m, 2H), 7.55-7.50 (m, 3H), $2.44(\mathrm{~s}, 3 \mathrm{H}) ;{ }^{13} \mathrm{C}-\mathrm{NMR}\left(100 \mathrm{MHz}, \mathrm{CDCl}_{3}\right) \delta 169.5$, $161.8,132.4,132.1,131.2,129.2,127.8,127.2,126.6$, 118.5, 112.3, 98.5, 94.4, 83.4, 10.8; LRMS (ESI) $\mathrm{m} / z$ (rel int): $285\left(\left[\mathrm{M}+\mathrm{H}^{+}, 92\right), 244\left(\left[\mathrm{M}-\mathrm{CH}_{2} \mathrm{CN}\right]^{+}, 32\right)\right.$; HRMS (ESI) $m / z$ calcd for $\mathrm{C}_{19} \mathrm{H}_{12} \mathrm{~N}_{2} \mathrm{O} 284.0950$, found 284.0950.

Synthesis of 3-Methyl-4-(4-nitrophenylethynyl)-5-phenylisoxazole (4j). 1-Ethynyl-4-nitrobenzene (3j) was used. The suspension was stirred for $19 \mathrm{~h}$ at rt. Column chromatography (40:1 hexane/EtOAc) afforded $\mathbf{4 j}$ (44 $\mathrm{mg}, 36 \%$ ) as a white solid, 5 (10 mg, 16\%) as a yellow solid, 6 (16.7 mg, $24 \%$ ) as a yellow liquid.

4j: mp $144-146{ }^{\circ} \mathrm{C}$ (from EtOAc); TLC: $R_{f} 0.15$ (14:1 hexane/EtOAc); ${ }^{1} \mathrm{H}-\mathrm{NMR}\left(400 \mathrm{MHz}, \mathrm{CDCl}_{3}\right) \delta 8.27$ (dt, $2 \mathrm{H}$, $J=6.8,2.0 \mathrm{~Hz}), 8.16-8.13(\mathrm{~m}, 2 \mathrm{H}), 7.69(\mathrm{dt}, 2 \mathrm{H}, J=6.8,2.0$ $\mathrm{Hz}), 7.54-7.52$ (m, 3H), 2.46 (s, 3H); ${ }^{13} \mathrm{C}-\mathrm{NMR}(100 \mathrm{MHz}$, $\left.\mathrm{CDCl}_{3}\right) \delta 169.7,161.8,147.5,132.4,131.3,129.7,129.2$, 127.2, 126.6, 124.0, 98.4, 94.2, 84.3, 10.8; LRMS (ESI) $\mathrm{m} / \mathrm{z}$ (rel int): $305\left([\mathrm{M}+\mathrm{H}]^{+}, 77\right), 264\left(\left[\mathrm{M}-\mathrm{CH}_{2} \mathrm{CN}\right]^{+}, 100\right)$; HRMS (ESI) $\mathrm{m} / \mathrm{z}$ calcd for $\mathrm{C}_{18} \mathrm{H}_{12} \mathrm{~F}_{2} \mathrm{O}_{3} 304.0848$, found 304.0848 .

Synthesis of 4-(3,4-Dichlorophenylethynyl)-3-methyl5-phenylisoxazole (4k). 1,2-dichloro-4-ethynylbenzene (3k) was used. The suspension was stirred for $17 \mathrm{~h}$ at $60{ }^{\circ} \mathrm{C}$. Column chromatography (40:1 hexane/EtOAc) afforded $\mathbf{4 k}$ (45.0 $\mathrm{mg}, 34 \%)$ as a yellow solid, $5(9.7 \mathrm{mg}, 15 \%)$ as a yellow solid, 6 (34.5 mg, 48\%) as a yellow liquid, and $7 \mathbf{k}$ (38.0 $\mathrm{mg}, 14 \%$ ) as a yellow solid.

4k: $\mathrm{mp} 135-137{ }^{\circ} \mathrm{C}$ (from EtOAc); TLC: $R_{f} 0.30$ (14:1 hexane/EtOAc); ${ }^{1} \mathrm{H}-\mathrm{NMR}\left(400 \mathrm{MHz}, \mathrm{CDCl}_{3}\right) \delta 8.14-8.12$ $(\mathrm{m}, 2 \mathrm{H}), 7.63(\mathrm{~d}, 1 \mathrm{H}, J=2.0 \mathrm{~Hz}), 7.54 ? 7.49(\mathrm{~m}, 3 \mathrm{H}), 7.47$ $(\mathrm{d}, 1 \mathrm{H}, J=8.4 \mathrm{~Hz}), 7.37(\mathrm{dd}, 1 \mathrm{H}, J=8.4,2.0 \mathrm{~Hz}), 2.43(\mathrm{~s}$, $3 \mathrm{H}) ;{ }^{13} \mathrm{C}-\mathrm{NMR}\left(100 \mathrm{MHz}, \mathrm{CDCl}_{3}\right) \delta 168.9,161.7,133.3$, $133.0,132.8,131.6,130.9,130.6,129.0,127.1,126.3$, 122.6, 98.5, 93.6, 80.8, 10.6; LRMS (ESI) $m / z$ (rel int): 328 $\left([\mathrm{M}+\mathrm{H}]^{+}, 100\right)$; HRMS (ESI) $m / z$ calcd for $\mathrm{C}_{18} \mathrm{H}_{11} \mathrm{Cl}_{2} \mathrm{NO}$ 327.0218, found 327.0218 .

7k: mp $194-196{ }^{\circ} \mathrm{C}$ (from EtOAc); TLC: $R_{f} 0.50$ (14:1 hexane/EtOAc); IR ( $\mathrm{KBr}$ film) 3405, 2306, 1655, 1261, 1026, 999, 827, 766, $669 \mathrm{~cm}^{-1}$; ${ }^{1} \mathrm{H}-\mathrm{NMR}\left(400 \mathrm{MHz}, \mathrm{CDCl}_{3}\right)$ $\delta 7.61(\mathrm{~d}, 2 \mathrm{H}, J=2.0 \mathrm{~Hz}), 7.43(\mathrm{~d}, 2 \mathrm{H}, J=8.4 \mathrm{~Hz}), 7.35$ (dd, $2 \mathrm{H}, J=8.4,2.0 \mathrm{~Hz}) ;{ }^{13} \mathrm{C}-\mathrm{NMR}\left(500 \mathrm{MHz}, \mathrm{CD}_{3} \mathrm{OD}\right) \delta 131.2$, $130.9,128.7,95.0,68.0,66.1,65.8,53.6$.

Synthesis of 3-Methyl-4-(4-methylphenylethynyl)-5phenylisoxazole (4I). 1-Ethynyl-4-methylbenzene (3I) and $\mathrm{K}_{3} \mathrm{PO}_{4}$ were used. The suspension was stirred for $19 \mathrm{~h}$ at $\mathrm{rt}$. Column chromatography (40:1 hexane/EtOAc) afforded of 41 (26.8 $\mathrm{mg}, 25 \%)$ as a yellow solid, 5 (32.2 $\mathrm{mg}, 51 \%)$ as a yellow solid, 6 (10.0 mg, 14\%) as a yellow liquid, and $7 \mathbf{l}$ (36.7 $\mathrm{mg}, 20 \%)$ as a yellow solid.

4l: $\mathrm{mp} 73-75{ }^{\circ} \mathrm{C}$ (from EtOAc); TLC: $R_{f} 0.20$ (14:1 hexane/EtOAc); ${ }^{1} \mathrm{H}-\mathrm{NMR}\left(400 \mathrm{MHz}, \mathrm{CDCl}_{3}\right) \delta 8.19-8.17$ $(\mathrm{m}, 2 \mathrm{H}), 7.52-7.47(\mathrm{~m}, 3 \mathrm{H}), 7.45(\mathrm{~d}, 2 \mathrm{H}, J=8.0 \mathrm{~Hz}), 7.20$ (d, $2 \mathrm{H}, J=8.0 \mathrm{~Hz}), 2.43$ (s, 3H), $2.40(\mathrm{~s}, 3 \mathrm{H}) ;{ }^{13} \mathrm{C}-\mathrm{NMR}$ 
$\left(100 \mathrm{MHz}, \mathrm{CDCl}_{3}\right) \delta 168.2,162.0,139.2,131.6,130.7$, $129.5,129.0,127.6,126.4,119.9,99.5,96.5,78.2,21.8$, 10.8; LRMS (ESI) $m / z$ (rel int): 274 ([M + H] $\left.]^{+}, 89\right), 233$ ([M $\left.-\mathrm{CH}_{2} \mathrm{CN}\right]^{+}, 100$ ); HRMS (ESI) $m / z$ calcd for $\mathrm{C}_{19} \mathrm{H}_{15} \mathrm{NO}$ 273.1154 , found 273.1158 .

7l: $\mathrm{mp} 140-142{ }^{\circ} \mathrm{C}$ (from EtOAc) (Lit., ${ }^{27} 137-138{ }^{\circ} \mathrm{C}$ ); TLC: $R_{f} 0.45$ (14:1 hexane/EtOAc); ${ }^{1} \mathrm{H}-\mathrm{NMR}(400 \mathrm{MHz}$, $\left.\mathrm{CDCl}_{3}\right) \delta$ 7.43-7.41 (m, 4H), 7.15-7.13 (m, 4H), $2.37(\mathrm{~s}$, $6 \mathrm{H}) ;{ }^{13} \mathrm{C}-\mathrm{NMR}\left(100 \mathrm{MHz}, \mathrm{CDCl}_{3}\right) \delta 139.7,132.6,129.4$, 119.0, 81.8, 73.7, 21.8; LRMS (ESI) $m / z$ (rel int): 231 ([M + $\mathrm{H}]^{+}, 100$ ); HRMS (ESI) $m / z$ calcd for $\mathrm{C}_{18} \mathrm{H}_{14}$ 230.1096, found 230.1096 .

\section{Results and Discussion}

Recently, $\alpha, \beta$-acetylenic oximes of hydroxylamine have been cyclized to the corresponding isoxazoles in the presence of gold catalyst. ${ }^{18}$ Thus, we initially thought that the $\alpha, \beta$-acetylenic oximes of hydroxylamine could serve as substrates for a one-pot cyclization-oxidative cross-coupling reaction. However, when 4-phenylbut-3-yn-2-one oxime (1b) was used as a substrate, it provided mainly isoxazole 5 from a cyclization-protodeauration and a trace of oxidative cross-coupling product 4a (Scheme 2). (Z)-4-Phenylbut-3yn-2-one oxime was equilibrated with $(E)$ at room temperature and cyclized into the corresponding isoxazole even without gold catalyst. This uncatalyzed cyclization was rapid and was a potential problem for one-pot cyclizationoxidative cross-coupling reaction because it could not generate gold-complexed intermediate $\mathbf{2}$. Therefore, we considered $O$-methyloximes as substrates, which were not cyclized spontaneously at room temperature and could be readily synthesized from commercially available ynones and methoxyamine hydrochloride in the presence of sodium acetate at room temperature using ethanol as the solvent. A mixture of $E-Z$ isomers sometimes resulted and the desired $Z$ isomer could be separated by column chromatography.

With the desired (Z)-4-phenylbut-3-yn-2-one $O$-methyloxime (1a) in hand, we began to investigate various gold catalysts and combinations with silver salts: $\left(\mathrm{PPh}_{3}\right) \mathrm{AuCl}$, $\left(\mathrm{PCy}_{3}\right) \mathrm{AuCl},(\mathrm{PhO})_{3} \mathrm{PAuCl}, \mathrm{AuCl}, \mathrm{AuCl}_{3},\left(\mathrm{PPh}_{3}\right) \mathrm{AuNTf}_{2}$, (IPr)AuCl (IPr $=N, N^{\prime}$-bis(2,6-diisopropylphenyl)imidazol2-ylidene), along with AgOTf, $\mathrm{AgNTf}_{2}$, or $\mathrm{AgSbF}_{6}$ (Table

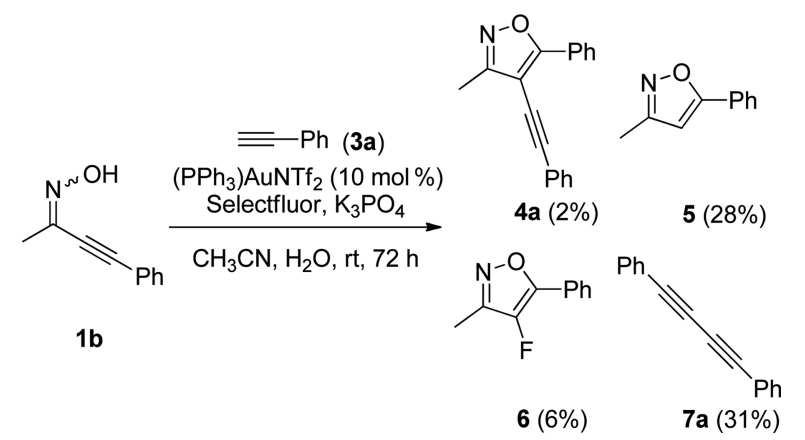

Scheme 2. Gold-catalyzed cyclization-oxidative alkynylation and cyclization-fluorination of oxime $\mathbf{1 b}$.
1). To our delight, most Au catalysts produced cyclizationoxidative cross-coupling product 4a except (IPr)AuCl (Table 1, entry 18). Under the standard reaction conditions, $\left(\mathrm{PPh}_{3}\right) \mathrm{AuCl}$ afforded $\mathbf{4 a}$ in $29 \%$ yield as well as cyclized isoxazole 5, fluorinated isoxazole $\mathbf{6}$, and alkyne dimer $\mathbf{7}$

Table 1. Optimization of gold-catalyzed cyclization-oxidative alkynylation and cyclization-fluorination of $(Z)$-2-alkynone $O$ methyloxime $\mathbf{1 a}^{a}$

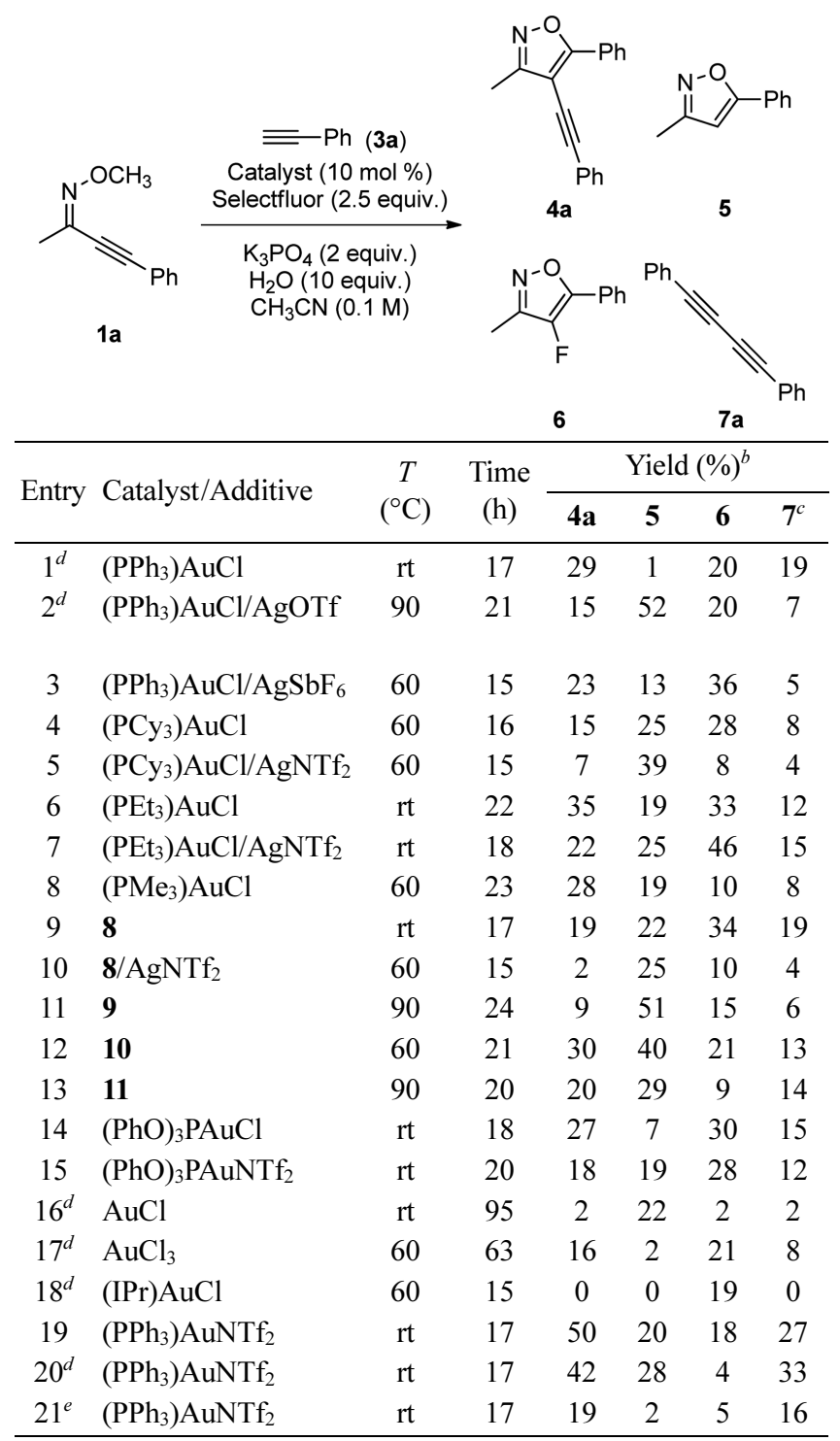

${ }^{a}$ Reaction conditions: 1a $(400 \mu \mathrm{mol}), \mathbf{3 a}(800 \mu \mathrm{mol})$, Au-catalyst $(40.0$ $\mu \mathrm{mol})$, Ag-salt $(40.0 \mu \mathrm{mol})$, Selectfluor $(1.00 \mathrm{mmol}), \mathrm{K}_{3} \mathrm{PO}_{4}(80.0$ $\mu \mathrm{mol}), \mathrm{H}_{2} \mathrm{O}(4.00 \mathrm{mmol}), \mathrm{CH}_{3} \mathrm{CN}(4 \mathrm{~mL}) .{ }^{b}$ Isolated yields. ${ }^{C}$ Isolated yields based on 3a. ${ }^{d} 600 \mu \mathrm{mol}$ of $\mathbf{3 a}$ was used. ${ }^{e} 1.20 \mathrm{mmol}$ of $\mathbf{3 a}$ was used.

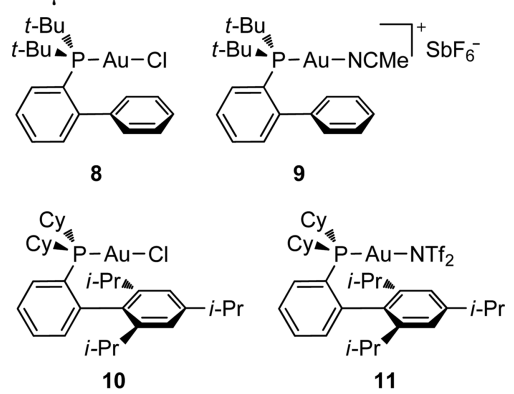


(Table 1, entry 1). Notably, no homocoupling product of $\mathbf{1 a}$ was observed. The addition of AgOTf or $\mathrm{AgSbF}_{6}$ made the reaction slower at room temperature and increased the formation of cyclized isoxazole 5 (entries 2 and 3). The gold(I) complex $\left(\mathrm{PEt}_{3}\right) \mathrm{AuCl}$ afforded $\mathbf{4 a}$ in $35 \%$ yield with increased formation of 5 (entry 6). Among the gold(I) complexes 8-11 bearing bulky biphenyl-based phosphine ligands, which have been shown to be excellent ligands for Pd-catalyzed reactions, ${ }^{19}$ only $\mathbf{1 0}$ yielded comparable results and produced $\mathbf{4 a}$ and $\mathbf{5}$ in $30 \%$ and $40 \%$ yields, respectively (entry 12). After extensive optimization studies, $\left(\mathrm{PPh}_{3}\right)$ AuNTf $_{2}(10 \mathrm{~mol} \%)$ was identified as the best catalyst for one-pot cyclization-oxidative cross-coupling reaction (entry 19). Under this condition, cross-coupling product $\mathbf{4 a}$ and fluorinated product 6 were isolated in $50 \%$ and $18 \%$ yield, respectively. Furthermore, when 2 equivalents of alkyne 3a were used, a better result was obtained than when 1.5 or 3 equivalents of alkyne 3a were used (entry $19 v s$. entries 20 and 21).

The role of $\mathrm{H}_{2} \mathrm{O}$ in gold-catalyzed reactions is still unclear and under investigation. Recently, it has been reported that the addition of $\mathrm{H}_{2} \mathrm{O}$ enhances the solubility of the reagents such as base and Selectfluor, which are poorly soluble in $\mathrm{CH}_{3} \mathrm{CN}^{20}$ Indeed, when the reaction was carried out in the presence of $4 \AA$ molecular sieves without the addition of $\mathrm{H}_{2} \mathrm{O}$, 4a was obtained in decreased yield (35\%, Scheme 3$)$. Using $\mathrm{MeOH}$ instead of $\mathrm{H}_{2} \mathrm{O}$ did not improve the yield of $\mathbf{4 a}$ (33\%, Scheme 3).

The base effect has also been extensively studied as shown in Table 2. Increasing the amount of potassium phosphate tribasic did not improve the yield of $\mathbf{4 a}$ (3 equiv., entry 2 ). Interestingly, the reactions of carbonate bases provided comparable results, except that of $\mathrm{Cs}_{2} \mathrm{CO}_{3}$, which was sluggish even at $60{ }^{\circ} \mathrm{C}$ and afforded 5 as a sole product in $28 \%$ yield (entries 4-9). Meanwhile, usage of amine bases such as $\mathrm{Et}_{3} \mathrm{~N}$ and 2,6-lutidine increased the formation of $\mathbf{5}$ instead of $\mathbf{4 a}$ (entries 12 and 13).

Selectfluor has been known as the best external oxidant for gold-catalyzed cross-coupling reactions. ${ }^{21}$ When the

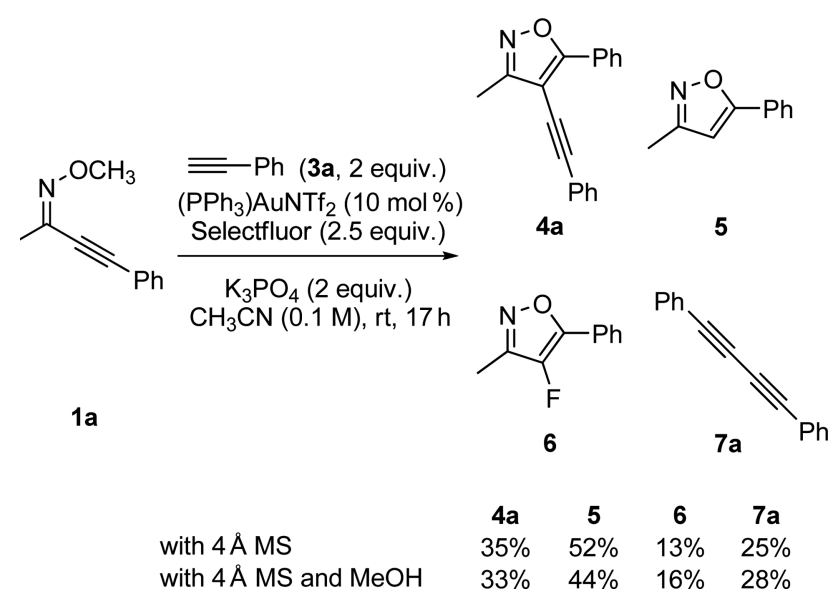

Scheme 3. Gold-catalyzed cyclization-oxidative alkynylation and cyclization-fluorination in the absence of $\mathrm{H}_{2} \mathrm{O}$.
Table 2. Base effect on the cyclization-oxidative alkynylation and cyclization-fluorination reactions of (Z)-2-alkynone $O$-methyloxime $1 \mathbf{a}^{a}$

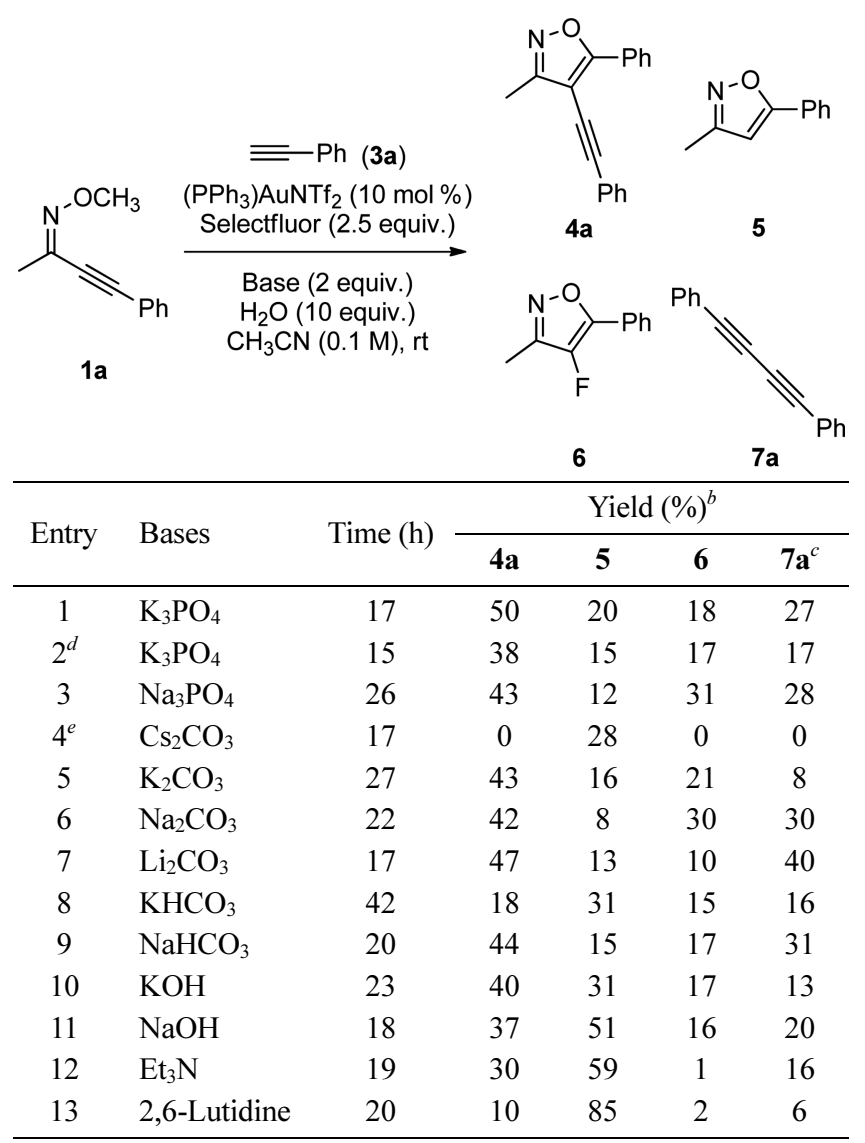

${ }^{a}$ Reaction conditions: 1a $(400 \mu \mathrm{mol}), 3 a(800 \mu \mathrm{mol})$, Au-catalyst $(40.0$ $\mu \mathrm{mol})$, Selectfluor $(1.00 \mathrm{mmol})$, Base $(800 \mu \mathrm{mol}), \mathrm{H}_{2} \mathrm{O}(4.00 \mathrm{mmol})$, $\mathrm{CH}_{3} \mathrm{CN}(4 \mathrm{~mL}) .{ }^{b}$ Isolated yields. "Isolated yields based on 3a. ${ }_{3}$ equivalents of base were used. ${ }^{e}$ The reaction was carried out at $60{ }^{\circ} \mathrm{C}$ and $72 \%$ of starting 1 a was recovered.

reaction was carried out in the presence of 1.5 equivalents of Selectfluor, 4a was isolated in $15 \%$ yield and $61 \%$ of starting (Z)-2-alkynone $O$-methyloxime 1a was recovered after 2 days (Table 3, entry 1 ). The best yield was observed with 2.5 equivalents of Selectfluor (Table 1, entry 19). Using more than 2.5 equivalents of Selectfluor decreased the yield of $\mathbf{4 a}$ from $50 \%$ to $48 \%$ (Table 3, entry 3 ). Meanwhile, when the amount of Selectfluor was increased from 1.5 to 3.0 equivalents, the formation of fluorinated isoxazole $\mathbf{6}$ increased from $3 \%$ to $20 \%$. As expected, other oxidants such as $\mathrm{PhI}(\mathrm{OAc})_{2}$, oxone, and $t$-BuOOH did not generate fluorinated isoxazole 6 (entries 4-7), neither did $N$-fluorobenzenesulfonimide (NFSI), even carrying fluorine source (entries 8 and 9). The reactions of these oxidants were very sluggish in the formation of $\mathbf{4 a}$ even at $80^{\circ} \mathrm{C}$. Interestingly, oxone afforded cyclized isoxazole $\mathbf{5}$ in $71 \%$ yield as a major product at $60{ }^{\circ} \mathrm{C}$ (entry 6).

Current cyclization-oxidative alkynylation and cyclizationfluorination reactions showed profound concentration effect and solvent effect on the yield of the desired product. Upon increasing the reaction concentration from 0.1 to $0.5 \mathrm{M}$ (Table 1, entry $19 v s$. Table 4, entry 1), the yield of $4 \mathbf{a}$ decreased 
Table 3. Oxidant effect on the cyclization-oxidative alkynylation and cyclization-fluorination reactions of $(Z)$-2-alkynone $O$-methyloxime 1a ${ }^{a}$

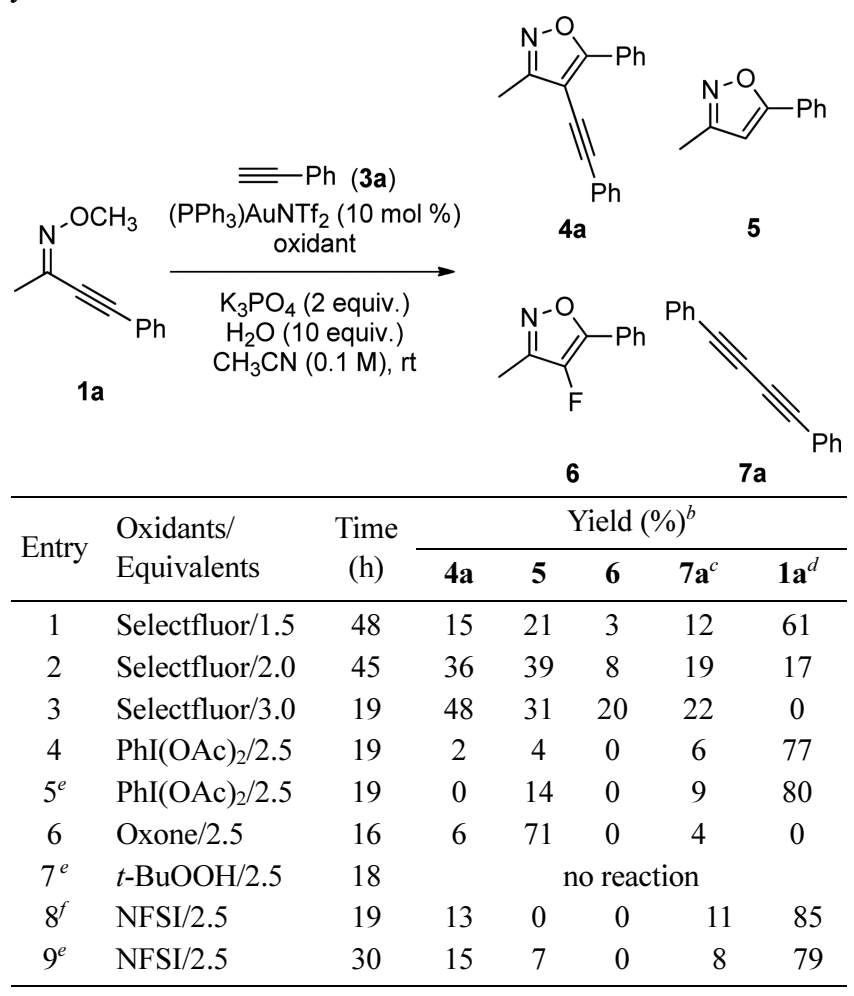

${ }^{a}$ Reaction conditions: 1a $(400 \mu \mathrm{mol})$, 3a $(800 \mu \mathrm{mol}),\left(\mathrm{PPh}_{3}\right) \mathrm{AuNTf}_{2}$ (40.0 $\mu \mathrm{mol})$, oxidant, $\mathrm{K}_{3} \mathrm{PO}_{4}(800 \mu \mathrm{mol}), \mathrm{H}_{2} \mathrm{O}(4.00 \mathrm{mmol}), \mathrm{CH}_{3} \mathrm{CN}(4$ $\mathrm{mL}) .{ }^{b}$ Isolated yields. ${ }^{c}$ Isolated yields based on $\mathbf{3 a} .{ }^{{ }} \%$ of recovered $1 \mathbf{a}$. ${ }^{e} 800^{\circ} \mathrm{C} .{ }^{f} 60{ }^{\circ} \mathrm{C}$

from $50 \%$ to $8 \%$, and $30 \%$ of starting (Z)-2-alkynone $O$ methyloxime 1a was recovered. In contrast, upon lowering the reaction concentration from 0.1 to $0.02 \mathrm{M}$ (Table 1 , entry 19 vs. Table 4, entry 2), 4a was isolated in 40\% yield after the extended reaction time $(93 \mathrm{~h})$. Alternative solvent such as DMF was less effective even at $80{ }^{\circ} \mathrm{C}$, leading to the formation of cyclized isoxazole 5 in $43 \%$ as a major product and $\mathbf{4 a}$ in $16 \%$ (entry 3 ). In less polar solvent 1,4-dioxane, the yield was far below the predictions: only $2 \%$ yield of $\mathbf{4 a}$ was obtained at $60{ }^{\circ} \mathrm{C}$ (entry 4). In particular, no conversion was observed in the relatively non-polar solvents, DCE and toluene (entries 5 and 6).

Under the standard reaction conditions, the reaction scope with respect to various alkyne coupling partners was probed as shown in Table 5 . The reaction proceeded smoothly with alkynes bearing electron-withdrawing groups on the phenyl ring (entries 1-10). The reaction with alkyne $\mathbf{3 b}$, which has $\mathrm{Cl}$ on the phenyl ring, led to $4 \mathbf{b}$ in $47 \%$ yield with the formation of 5, $\mathbf{6}$ and $\mathbf{7}$ at ambient temperature (entry 1). On the other hand, the reactions with alkynes bearing electrondonating groups on the phenyl ring were sluggish and did not provide the coupling products (entries 12 and 13), except for the reaction of alkyne $\mathbf{3 l}$ (entry 11). Instead, the reactions with these electron-rich alkynes afforded cycloisomerized isoxazole $\mathbf{5}$ as a major product. Current observations are consistent with those of the Sonogashira cross-coupling
Table 4. Solvent effect on the cyclization-oxidative alkynylation and cyclization-fluorination reactions of $(Z)$-2-alkynone $O$-methyloxime $1 \mathbf{a}^{a}$

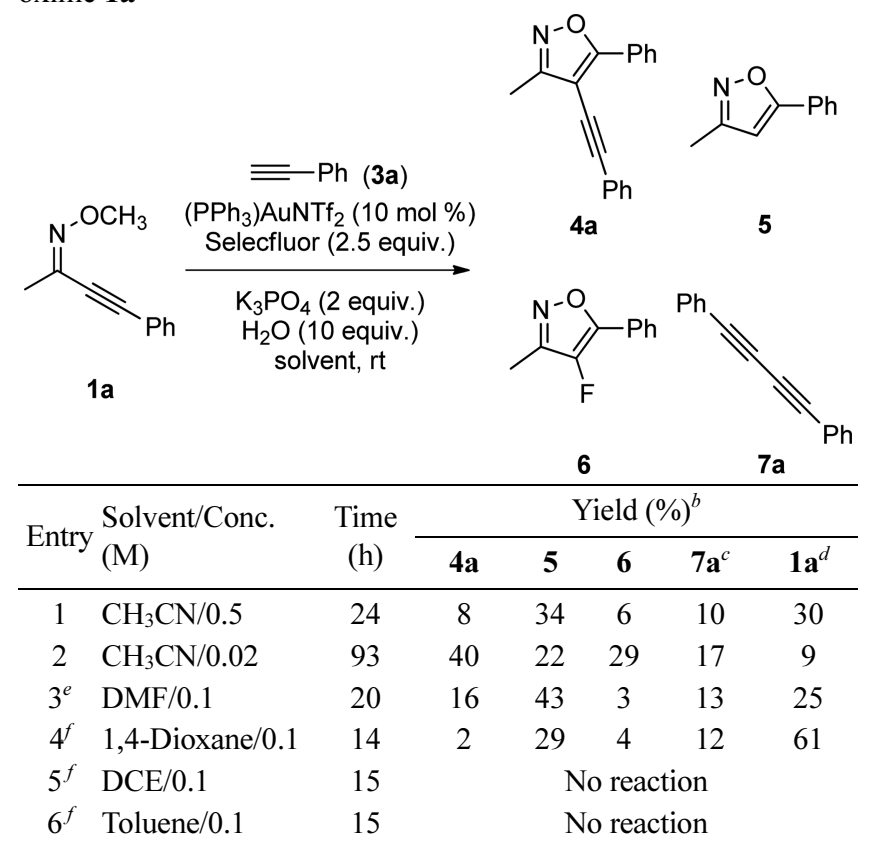

${ }^{a \text { Reaction conditions: 1a }(400 \mu \mathrm{mol}), 3 a(800 \mu \mathrm{mol}),\left(\mathrm{PPh}_{3}\right) \mathrm{AuNTf}_{2}}$ $(40.0 \mu \mathrm{mol})$, Selectfluor $(1.00 \mathrm{mmol}), \mathrm{K}_{3} \mathrm{PO}_{4}(800 \mu \mathrm{mol}), \mathrm{H}_{2} \mathrm{O}(4.00$ mmol), Solvent. ${ }^{b}$ Isolated yields. ${ }^{C}$ Isolated yields based on 3a. ${ }^{d} \%$ of recovered 1a. ${ }^{e} 80{ }^{\circ} \mathrm{C} .{ }^{f} 60{ }^{\circ} \mathrm{C}$

process: The reaction proceeds more rapidly with more electron-poor alkynes. ${ }^{22} \mathrm{~K}_{3} \mathrm{PO}_{4}$ is supposed to abstract the acetylenic proton of the terminal alkyne, thus forming a gold-acetylide species (vide infra) ${ }^{23}$ It should be pointed out that the employed bases are usually not basic enough to deprotonate the alkyne in order to generate the anionic nucleophile leading to the gold-acetylide species. Therefore, electron-withdrawing substituents on the phenyl ring that make the alkyne proton more acidic would lead to easier abstraction of the acetylenic proton.

To discriminate the cyclization-oxidative alkynylation mechanism from the alkynylation through direct $\mathrm{C}-\mathrm{H}$ activation of in situ formed isoxazole $\mathbf{5}$, the isoxazole $\mathbf{5}$ was prepared independently and treated with $\left(\mathrm{PPh}_{3}\right) \mathrm{AuNTf}_{2}(10$ mol \%) in the presence of Selectfluor, $\mathrm{K}_{3} \mathrm{PO}_{4}$, and $\mathrm{H}_{2} \mathrm{O}$ (Scheme 4). This experiment led to recovery of the starting material $\mathbf{5}$ and formation of alkyne dimer 7 with no detectable trace of the alkynylation product $\mathbf{4 a}$ and fluorinated isoxazole 6, which suggests that the alkynylation mechanism does not operate through direct $\mathrm{C}-\mathrm{H}$ activation of isoxazole 5. Therefore, the product $\mathbf{4 a}$ would be formed through oxidative coupling before the protodeauration of the gold(I) complex.

On the basis of previous literature and our observation, two plausible mechanistic pathways can be suggested for the gold-catalyzed cyclization-oxidative alkynylation of ( $Z$ )-2alkynone $O$-methyloxime (Scheme 5). Initial coordination of the gold(I) catalyst $\mathbf{A}$ to the terminal alkyne $\mathbf{3 a}$ would form a $\pi$-coordinated alkyne adduct $\mathbf{B}$, which transforms into the $\sigma$ - 
Table 5. Synthesis of 3,4,5-trisubstituted isoxazoles by gold-catalyzed cascade cyclization-oxidative alkynylation and cyclizationfluorination of (Z)-2-alkynone $O$-methyloximes ${ }^{a}$

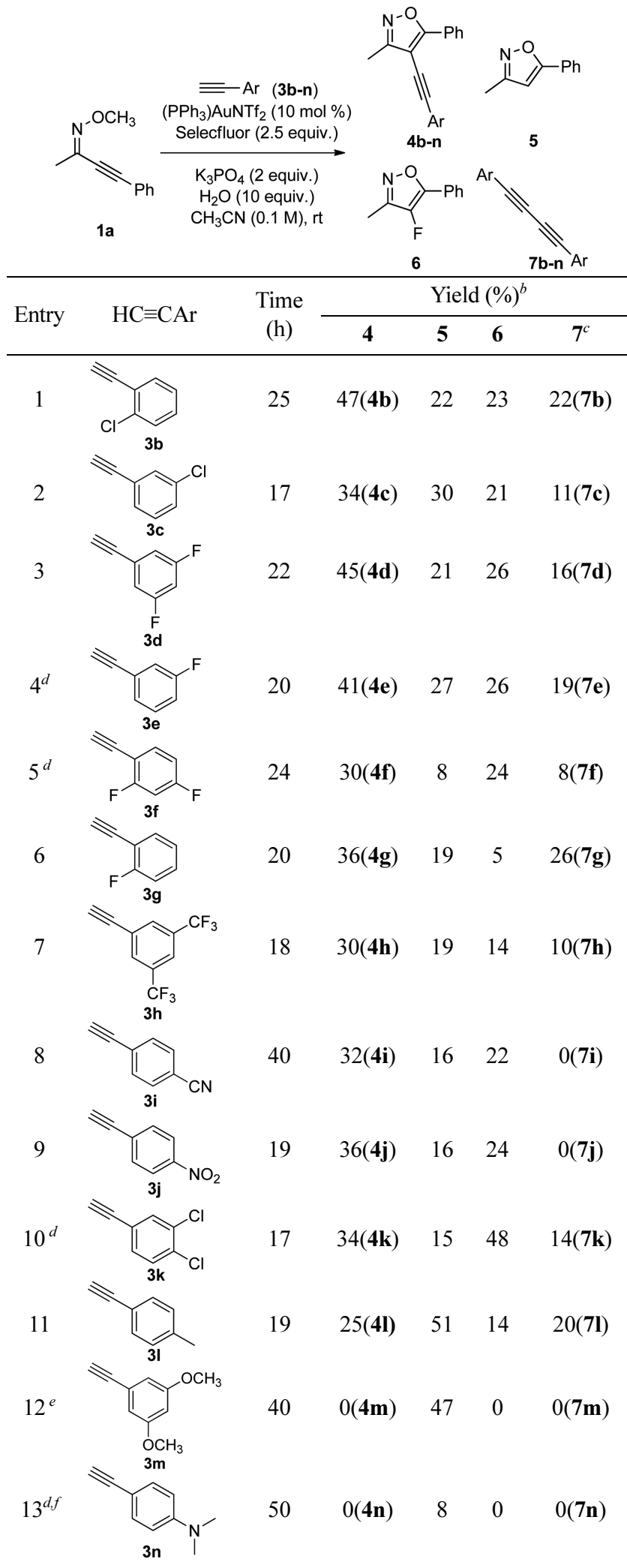

${ }^{a}$ Reaction conditions: 1a $(400 \mu \mathrm{mol}), \mathbf{3 b}-\mathbf{n}(800 \mu \mathrm{mol}),\left(\mathrm{PPh}_{3}\right) \mathrm{AuNTf}_{2}$ $(40.0 \mu \mathrm{mol})$, Selectfluor $(1.00 \mathrm{mmol}), \mathrm{K}_{3} \mathrm{PO}_{4}(800 \mu \mathrm{mol}), \mathrm{H}_{2} \mathrm{O}(4.00 \mathrm{mmol})$, $\mathrm{CH}_{3} \mathrm{CN}(4 \mathrm{~mL}) .{ }^{b}$ Isolated yields. ${ }^{c}$ Isolated yields based on $\mathbf{3 b}-\mathbf{n} .{ }^{d} 60{ }^{\circ} \mathrm{C}$. ${ }^{e} 45 \%$ of staring 1 a was recovered. ${ }^{f} 80 \%$ of staring 1 a was recovered.

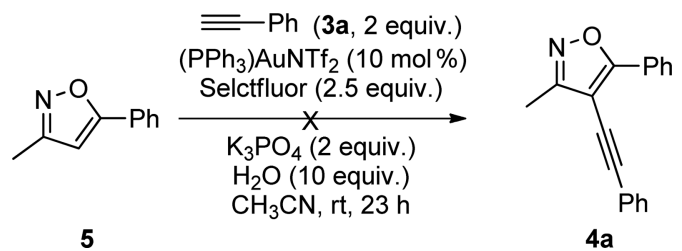

Scheme 4. Gold-catalyzed alkynylation of isoxazole 5 .

acetylide gold(I) complex $\mathbf{C}$ in the presence of base. Then, oxidation by Selectfluor would afford the cationic gold(III) intermediate $\mathbf{D}$, which reacts with the second molecule of $\mathbf{C}$ and gives the homo-coupled product 7 . This mechanism has been well documented recently. ${ }^{24}$ On the other hand, the coordination of gold(III) intermediate $\mathbf{D}$ to the alkyne of $O$ methyloxime 1a and subsequent cyclization would lead to the gold(III) complex $\mathbf{E}$ bearing both isoxazole and alkyne substituents; E readily decomposes to yield cross-coupled product 4a and the cationic gold(I) catalyst $\mathbf{A}$ after reductive elimination. In the other possible mechanistic pathway, the gold(I)-catalyzed cyclization of $O$-methyloxime 1a may occur before the formation of $\sigma$-acetylide gold(I) complex $\mathbf{C}$. The gold(I) complex $\mathbf{G}$, which is formed after the demethylation of the cyclized gold(I) complex $\mathbf{F}$, may give the protodeauration product 5. Otherwise, Selectfluor would oxidize the gold(I) complex $\mathbf{G}$ to the cationic gold(III) intermediate $\mathbf{H}$, the reductive elimination of which would afford the fluorinated isoxazole 6, or the fluoride-acetylide ligand exchange of which would give the gold(III) complex E. As mentioned previously, $\mathbf{E}$ undergoes reductive elimination to provide cross-coupled product $4 \mathbf{a}$ and to regenerate the cationic gold(I) catalyst $\mathbf{A}$. This suggested mechanism involves a redox $\mathrm{Au}(\mathrm{I}) / \mathrm{Au}(\mathrm{III})$ catalytic cycle mediated by the external oxidant Selectfluor.

\section{Conclusion}

In conclusion, we have developed a novel one-pot method to synthesize trisubstituted isoxazoles from (Z)-2-alkynone $O$-methyloxime. This gold-catalyzed cascade reaction proceeds under mild conditions (room temperature) via the intermolecular addition of the oxime oxygen atom to the tethered triple bond and subsequent alkynylation or fluorination. Mechanistically, gold-catalyzed cycloisomerization has been combined with the gold-catalyzed oxidative crosscoupling or fluorination process. The current synthetic method is straightforward and represents a typical pot-economical synthesis in that no intermediate purification operations are needed. This is the first example to synthesize various alkynyl isoxazoles from structurally simple $O$-methyloxime in a tandem manner, and offers the efficient construction of functionalized isoxazoles.

Acknowledgments. This research was supported by the Basic Science Research Program through the National Research Foundation of Korea (NRF) funded by the Ministry of Education, Science and Technology (NRF- 


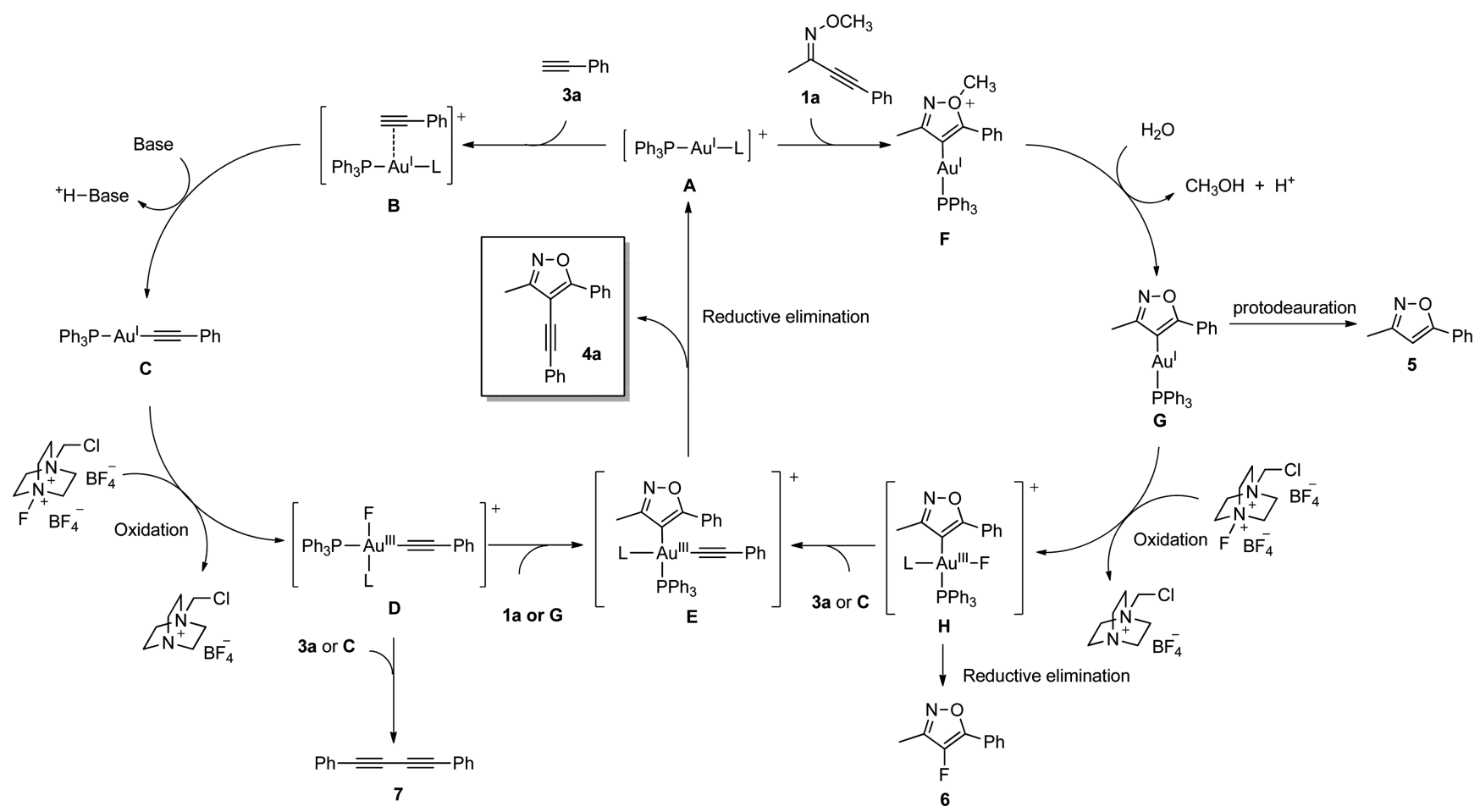

Scheme 5. Plausible mechanism for gold-catalyzed cyclization-oxidative alkynylation of (Z)-2-alkynone $O$-methyloxime.

2012R1A2A2A01011831).

\section{References}

1. Pinho, E. M.; Teresa, M. V. D. Curr. Org. Chem. 2005, 9, 925.

2. Daidone, G.; Raffa, D.; Maggio, B.; Plescia, F.; Cutuli, V. M. C.; Mangano, N. G.; Caruso, A. Arch. Pharm. 1999, 332, 50.

3. Cali, P.; Nærum, L.; Mukhija, S.; Hjelmencrantz, A. Bioorg. Med. Chem. Lett. 2004, 14, 5997.

4. Liu, J.; Yu, L.-F.; Eaton, J. B.; Caldarone, B.; Cavino, K.; Ruiz, C.; Terry, M.; Fedolak, A.; Wang, D.; Ghavami, A.; Lowe, D. A.; Brunner, D.; Lukas, R. J.; Kozikowski, A. P. J. Med. Chem. 2011, $54,7280$.

5. Kumbhare, R. M.; Kosurkar, U. B.; Ramaiah, M. J.; Dadmal, T. L.; Pushpavalli, S. N. C. V. L.; Pal-Bhadra, M. Bioorg. Med. Chem. Lett. 2012, 22, 5424.

6. Bandiera, T.; Grünanger, P.; Albini, F. M. J. Heterocycl. Chem. 1992, 29, 1423

7. Cuadrado, P.; Gonzalez-Nogal, A. M.; Valero, R. Tetrahedron 2002, 58, 4975 .

8. Heaney, F. Eur. J. Org. Chem. 2012, 3043.

9. Waldo, J. P.; Mehta, S.; Neuenswander, B.; Lushington, G. H.; Larock, R. C. J. Comb. Chem. 2008, 10, 658.

10. Moore, J. E.; Davies, M. W.; Goodenough, K. M.; Wybrow, R. A. J.; York, M.; Johnson, C. N.; Harrity, J. P. A. Tetrahedron 2005, $61,6707$.

11. Denmark, S. E.; Kallemeyn, J. M. J. Org. Chem. 2005, 70, 2839.

12. Trost, B. M. Angew. Chem. Int. Ed. 1995, 34, 259.

13. Clarke, P. A.; Santos, S.; Martin, W. H. C. Green Chem. 2007, 9 , 438.

14. Grecian, S.; Fokin, V. V. Angew. Chem. 2008, 120, 8409 and references therein.

15. (a) Ueda, M.; Sugita, S.; Sato, A.; Miyoshi, T.; Miyata, O. J. Org. Chem. 2012, 77, 9344. (b) She, Z.; Niu, D.; Chen, L.; Gunawan, M. A.; Shanja, X.; Hersh, W. H.; Chen, Y. J. Org. Chem. 2012, 77, 3627. (c) Gayon, E.; Quinonero, O.; Lemouzy, S.; Vrancken, E.;
Campagne, J.-M. Org. Lett. 2011, 13, 6418. (d) Ueda, M.; Sato, A.; Ikeda, Y.; Miyoshi, T.; Naito, T.; Miyata, O. Org. Lett. 2010, $12,2594$.

16. For selected reviews on gold catalysis, see: (a) Sengupta, S.; Shi, X. ChemCatChem. 2010, 2, 609. (b) Shapiro, N. D.; Toste, F. D. Synlett 2010, 675. (c) Muzart, J. Tetrahedron 2008, 64, 5815. (d) Li, Z.; Brouwer, C.; He, C. Chem. Rev. 2008, 108, 3239. (e) Hashmi, A. S. K. Chem. Rev. 2007, 107, 3180. (f) Gorin, D. J.; Toste, F. D. Nature 2007, 446, 395. (g) Hoffmann-Röder, A.; Krause, N. Org. Biomol. Chem. 2005, 3, 387. (h) Jiménez-Núñez, E.; Echavarren, A. M. Chem. Rev. 2008, 108, 3326. (i) Gorin, D. J.; Sherry, B. D.; Toste, F. D. Chem. Rev. 2008, 108, 3351. (j) Skouta, R.; Li, C.-J. Tetrahedron 2008, 64, 4917. (k) Widenhoefer, R. A. Chem. Eur. J. 2008, 14, 5382. (1) Fürstner, A. Chem. Soc. Rev. 2009, 38, 3208.

17. (a) Zhang, G.; Cui, L.; Wang, Y.; Zhang, L. J. Am. Chem. Soc. 2010, 132, 1474. (b) Melhado, A. D.; Brenzovich, W. E., Jr.; Lackner, A. D.; Toste, F. D. J. Am. Chem. Soc. 2010, 132, 8885 . (c) Brenzovich, W. E., Jr.; Benitez, D.; Lackner, A. D.; Shunatona, H. P.; Tkatchouk, E.; Goddard, W. A., III.; Toste, F. D. Angew. Chem., Int. Ed. 2010, 49, 5519. (d) Hopkinson, M. N.; Ross, J. E.; Giuffredi, G. T.; Gee, A. D.; Gouverneur, V. Org. Lett. 2010, 12, 4904. (e) Peng, Y.; Cui, L.; Zhang, G..; Zhang, L. J. Am. Chem. Soc. 2009, 131, 5062. (f) Zhang, G.; Peng, Y.; Cui, L.; Zhang, L. Angew. Chem., Int. Ed. 2009, 48, 3112.

18. Praveen, C.; Kalyanasundaram, A.; Perumal, P. T. Synlett 2010 , 777.

19. (a) Barder, T. E.; Buchwald, S. L. J. Am. Chem. Soc. 2007, 129, 5096. (b) Barder, T. E.; Walker, S. D.; Martinelli, J. R.; Buchwald, S. L. J. Am. Chem. Soc. 2005, 127, 4685. (c) Walker, S. D.; Barder, T. E.; Martinelli, J. R.; Buchwald, S. L. Angew. Chem., Int. Ed. 2004, 43, 1871. (d) Strieter, E. R.; Blackmond, D. G.; Buchwald, S. L. J. Am. Chem. Soc. 2003, 125, 13978. (e) Kaye, S.; Fox, J. M.; Hicks, F. A.; Buchwald, S. L. Adv. Synth. Catal. 2001, 343, 789 .

20. Zhang, G.; Luo, Y.; Wang, Y.; Zhang, L. Angew. Chem. Int. Ed. 2011, 50,4450 . 
21. (a) Nyffeler, P. T.; Durón, S. G.; Burkart, M. D.; Vincent, S. P.; Wong, C.-H. Angew. Chem. Int. Ed. 2005, 44, 192. (b) Stavber, S.; Zupan, M. Acta Chim. Slov. 2005, 52, 13.

22. Chinchilla, R.; Nájera, C. Chem. Rev. 2007, 107, 874.

23. Qian, D.; Zhang, J. Beilstein J. Org. Chem. 2011, 7, 808.

24. Leyva-Pérez, A.; Doménech, A.; Al-Resayes, S. I.; Corma, A. ACS Catal. 2012, 2, 121.

25. Valizadeh, H.; Amiri, M.; Gholipur, H. J. Heterocyclic Chem. 2009, 46, 108.
26. Merkul, E.; Urselmann, D.; Müller, T. J. J. Eur. J. Org. Chem. 2011, 238.

27. Chen, S.-N.; Wu, W.-Y.; Tsai, F.-Y. Green Chem. 2009, 11, 269.

28. Zhou, L.; Zhan, H.-Y.; Liu, H.-L.; Jiang, H.-F. Chin. J. Chem. 2007, 25, 1413.

29. Stefani, H. A.; Pena, J. M.; Zukerman-Schpector, J.; Tiekink, E. R. T. J. Braz. Chem. Soc. 2011, 22, 1439.

30. Kurita, T.; Abe, M.; Maegawa, T.; Monguchi, Y.; Sajiki, H. Synlett. 2007, 16, 2521. 